\title{
ANALYSIS OF DIRECT SEGREGATED BOUNDARY-DOMAIN INTEGRAL EQUATIONS FOR VARIABLE-COEFFICIENT MIXED BVPS IN EXTERIOR DOMAINS
}

\author{
O. Chkadua \\ A. Razmadze Mathematical Institute, I. Javakhishvili Tbilisi State University, \\ 2, University St., Tbilisi 0186, Georgia \\ chkadua@rmi.acnet.ge \\ S.E. Mikhailov* \\ Dept. of Mathematics, Brunel University London, \\ Uxbridge, UB8 3PH, United Kingdom \\ sergey.mikhailov@brunel.ac.uk \\ D. Natroshvili \\ Department of Mathematics, Georgian Technical University, \\ 77, M. Kostava St., Tbilisi 0175, Georgia \\ natrosh@hotmail.com
}

\begin{abstract}
Direct segregated systems of boundary-domain integral equations are formulated for the mixed (Dirichlet-Neumann) boundary value problems for a scalar second order divergent elliptic partial differential equation with a variable coefficient in an exterior three-dimensional domain. The boundary-domain integral equation system equivalence to the original boundary value problems and the Fredholm properties and invertibility of the corresponding boundary-domain integral operators are analyzed in weighted Sobolev spaces suitable for infinite domains. This analysis is based on the corresponding properties of the BVPs in weighted Sobolev spaces that are proved as well.
\end{abstract}

Keywords: Partial differential equation; variable coefficient; mixed problem; parametrix; Levi function; boundary-domain integral equations; unbounded domain; weighted Sobolev spaces.

Mathematics Subject Classification 2010: 35J25, 31B10, 45P05, 45A05, 47G10, 47G40

\section{Introduction}

Partial Differential Equations (PDEs) with variable coefficients often arise in mathematical modelling of inhomogeneous media (e.g. functionally graded materials or materials with damage induced inhomogeneity) in solid mechanics, electromagnetics, thermoconductivity, fluid flows trough porous media, and other areas of physics and engineering.

Generally, explicit fundamental solutions are not available if the PDE coefficients are not constant, preventing reduction of Boundary Value Problems (BVPs) for such PDEs

${ }^{*}$ Corresponding author 
to explicit boundary integral equations to be effectively solved numerically. Nevertheless, for a rather wide class of variable-coefficient PDEs it is possible to use instead an explicit parametrix (Levi function) associated with a fundamental solution of corresponding frozencoefficient PDEs, and reduce BVPs for such PDEs in interior domains to systems of Boundary-Domain Integral Equations (BDIEs) for further numerical solution of the latter, see e.g. $[2,4,15,20,16,17]$ and references therein.

Our main goal here is to show that the mixed problems with variable coefficients in exterior domains can be reduced to some systems of BDIEs and investigate equivalence of the reduction and invertibility of the corresponding boundary-domain integral operators in the weighted Sobolev spaces (that are more suitable for exterior domains than the standard Sobolev spaces). To do this, we extend to exterior domains and weighted spaces the methods developed in [2] for interior domains and standard Sobolev (Bessel potential) spaces.

The BDIE analysis heavily relies on the properties of the corresponding boundary value problems. The variable-coefficient BVPs in bounded domains are well studied nowadays, see e.g. $[12,9,14]$. Employing the variational methods and Lax-Milgram lemma, the uniqueness and solvability in the weighted Sobolev spaces for general divergent-form elliptic equations in $\mathbb{R}^{n}$ were proved by $[24,11]$ and for the Dirichlet and Neumann problems for the Poisson equation in exterior domains with compact boundary by $[22,8,13,7,6,23]$. These methods are extended here to analysis of unique solvability of variable-coefficient BVPs in exterior domains.

The analysis of the BDIEs is not only an interesting and challenging mathematical problem on its own right but is also useful for the BDIE discretisation and numerical solution to obtain by this way a numerical solution of the associated BVP. Although the BDIE numerical applications are beyond the scope of this paper, they are the subject of other publications, see e.g. $[29,30,28,25,15,20,26,19,10]$.

The paper is arranged as follows. Section 2 describes some weighted Sobolev spaces, the considered partial differential operator and the associated weak definition of the co-normal derivative. Section 3 presents the boundary value problems, which unique solvability is obtained in Section 8 (Appendix). Section 4 introduces parametrix and parametrix-based volume and boundary potentials and describes their properties in the weighted Sobolev spaces. In Section 5, the mixed BVP is reduced to four different segregated BDIE systems, which equivalence to the mixed BVP is analyzed in Section 6. In Section 7, the Fredholm properties and invertibility of the left hand side operators are proved in the appropriate Sobolev spaces.

\section{Basic Notations and Spaces}

Let $\Omega=\Omega^{+}$be an unbounded (exterior) open three-dimensional region of $\mathbb{R}^{3}$ such that $\Omega^{-}:=\mathbb{R}^{3} \backslash \bar{\Omega}$ is a bounded open domain. For simplicity, we assume that the boundary $\partial \Omega=\partial \Omega^{-}$is a simply connected, compact, infinitely smooth surface.

We consider below some boundary-domain integral equation systems associated with a mixed BVP for the following scalar elliptic differential equation

$$
A u(x):=A\left(x, \partial_{x}\right) u(x):=\sum_{i=1}^{3} \frac{\partial}{\partial x_{i}}\left(a(x) \frac{\partial u(x)}{\partial x_{i}}\right)=f(x), \quad x \in \Omega,
$$

where $u$ is an unknown function, while $a(x)>0$ and $f$ are given functions in $\Omega$.

In what follows, $H^{s}(\Omega)=H_{2}^{s}(\Omega), H^{s}(\partial \Omega)=H_{2}^{s}(\partial \Omega)$ denote the Bessel potential spaces (coinciding with the Sobolev-Slobodetski spaces if $s \geq 0$ ), $H_{\partial \Omega}^{s}:=\{g: g \in$ $H^{s}\left(\mathbb{R}^{3}\right)$, supp $\left.g \subset \partial \Omega\right\}$. For an open set $\Omega$, we, as usual, denote $\mathcal{D}(\Omega)=C_{\text {comp }}^{\infty}(\Omega)$ 
endowed with sequential continuity, $\mathcal{D}^{*}(\Omega)$ is the Schwartz space of sequentially continuous functionals on $\mathcal{D}(\Omega)$, while $\mathcal{D}(\bar{\Omega})$ is the set of restrictions on $\bar{\Omega}$ of functions from $\mathcal{D}\left(\mathbb{R}^{3}\right)$. We also denote $\widetilde{H}^{s}\left(S_{1}\right)=\left\{g: g \in H^{s}(S)\right.$, supp $\left.g \subset \overline{S_{1}}\right\}, H^{s}\left(S_{1}\right)=\left\{r_{S_{1}} g: g \in H^{s}(S)\right\}$, where $S_{1}$ is a proper submanifold of a closed surface $S$ and $r_{S_{1}}$ is the restriction operator on $S_{1}$.

To make the boundary-value problems for (2.1) in infinite domains uniquely solvable, we will use weighted Sobolev spaces (see e.g. $[11,22,8,13,7,6,23])$. Let $\partial_{j}=\partial_{x_{j}}:=\partial / \partial x_{j}$ $(j=1,2,3), \nabla=\partial_{x}=\left(\partial_{x_{1}}, \partial_{x_{2}}, \partial_{x_{3}}\right)$. Let $\rho(x):=\left(1+|x|^{2}\right)^{1 / 2}$ be the weight function,

$$
L_{2}\left(\rho^{-1} ; \Omega\right):=\left\{g: \rho^{-1} g \in L_{2}(\Omega)\right\}
$$

be the weighted Lebesgue space and $\mathcal{H}^{1}(\Omega)$ be the weighted Sobolev (Beppo-Levi) space,

$$
\begin{array}{r}
\mathcal{H}^{1}(\Omega):=\left\{g \in L_{2}\left(\rho^{-1} ; \Omega\right): \nabla g \in L_{2}(\Omega)\right\}, \\
\|g\|_{\mathcal{H}^{1}(\Omega)}^{2}:=\left\|\rho^{-1} g\right\|_{L_{2}(\Omega)}^{2}+\|\nabla g\|_{L_{2}(\Omega)}^{2} .
\end{array}
$$

Using the corresponding property for the space $H^{1}(\Omega)$, it is easy to prove that $\mathcal{D}(\bar{\Omega})$ is dense in $\mathcal{H}^{1}(\Omega)$, cf. [11, Theorem I.1], [7, Theorem 2.2]. If $\Omega$ is unbounded, then the seminorm

$$
|g|_{\mathcal{H}^{1}(\Omega)}:=\|\nabla g\|_{L_{2}(\Omega)}
$$

is equivalent to the norm $\|g\|_{\mathcal{H}^{1}(\Omega)}$ in $\mathcal{H}^{1}(\Omega)$, see e.g. [6, Ch. XI, Part B, §1]. If $\Omega^{-}$ is bounded, then $\mathcal{H}^{1}\left(\Omega^{-}\right)=H^{1}\left(\Omega^{-}\right)$. If $\Omega^{\prime}$ is a bounded subdomain of an unbounded domain $\Omega$ and $g \in \mathcal{H}^{1}(\Omega)$, then $g \in H^{1}\left(\Omega^{\prime}\right)$. More general weighted spaces for unbounded domains can be found e.g. in $[1,23]$ and references therein.

Let us define as $\widetilde{\mathcal{H}}^{1}(\Omega)$ a completion of $\mathcal{D}(\Omega)$ in $\mathcal{H}^{1}\left(\mathbb{R}^{3}\right)$, while $\widetilde{\mathcal{H}}^{-1}(\Omega):=\left[\mathcal{H}^{1}(\Omega)\right]^{*}$, $\mathcal{H}^{-1}(\Omega):=\left[\widetilde{\mathcal{H}}^{1}(\Omega)\right]^{*}$ are the corresponding dual spaces and $L_{2}(\rho ; \Omega):=\left\{g: \rho g \in L_{2}(\Omega)\right\}$. Evidently $L_{2}(\rho ; \Omega) \subset \mathcal{H}^{-1}(\Omega)$. Any distribution $g \in \widetilde{\mathcal{H}}^{-1}(\Omega)$ has a representation $g=\sum_{i=1}^{3} \partial_{i} g_{i}+g^{0}$, where $g_{i} \in L_{2}\left(\mathbb{R}^{3}\right)$ and are zero outside $\Omega, g^{0} \in L_{2}(\rho ; \Omega)$, cf. ansatz (2.5.129) in [23]. This implies that $\mathcal{D}(\Omega)$ is dense in $\widetilde{\mathcal{H}}^{-1}(\Omega)$ and $\mathcal{D}\left(\mathbb{R}^{3}\right)$ is dense in $\mathcal{H}^{-1}\left(\mathbb{R}^{3}\right)$.

The operator $A$ acting on $u \in \mathcal{H}^{1}(\Omega)$ is well defined in the distributional sense for $a \in L_{\infty}(\Omega)$ as

$$
\langle A u, v\rangle_{\Omega}:=-\langle a \nabla u, \nabla v\rangle_{\Omega}=-\mathcal{E}(u, v)
$$

for any $v \in \mathcal{D}(\Omega)$, where

$$
\mathcal{E}(u, v):=\int_{\Omega} E(u, v)(x) d x, \quad E(u, v)(x):=\nabla v(x) \cdot a(x) \nabla u(x) .
$$

Since the bilinear functional $\mathcal{E}(u, v): \mathcal{H}^{1}(\Omega) \times \widetilde{\mathcal{H}}^{1} \rightarrow \mathbb{R}$ is bounded, then by density of $\mathcal{D}(\Omega)$ in $\widetilde{\mathcal{H}}^{1}(\Omega)$, the linear operator $A: \mathcal{H}^{1}(\Omega) \rightarrow \mathcal{H}^{-1}(\Omega)$, defined by expression (2.4) for any $v \in \widetilde{\mathcal{H}}^{1}(\Omega)$, is continuous and gives the weak form of the operator $A$ from (2.1).

From the trace theorem (see, e.g., [12]) for $u \in H^{1}(\Omega)$ it follows that if $u \in \mathcal{H}^{1}\left(\Omega^{ \pm}\right)$, then $\gamma^{ \pm} u \in H^{\frac{1}{2}}(\partial \Omega)$, where $\gamma^{ \pm}=\gamma_{\partial \Omega}^{ \pm}$are the trace operators on $\partial \Omega$ from $\Omega^{ \pm}$. We will use $\gamma u$ for $\gamma^{ \pm} u$ if $\gamma^{+} u=\gamma^{-} u$. We will use also notations $u^{ \pm}$for the traces $\gamma^{ \pm} u$, when this will cause no confusion.

Unless said otherwise we henceforth assume that there are some constants $a_{0}, a_{1}$ such that

$$
a \in L_{\infty}\left(\mathbb{R}^{3}\right) \text { and } 0<a_{0}<a(x)<a_{1}<\infty \quad \text { for a.e. } x \in \mathbb{R}^{3} .
$$


For $u \in \mathcal{H}^{1}(\Omega)$ (as well as for $u \in H^{1}(\Omega)$ ) the co-normal derivative operators $a \partial_{n} u$ on $\partial \Omega$ may not exist in the classical (trace) sense. However for the linear operator $A$, we introduce the space, cf. [8],

$\mathcal{H}^{1,0}(\Omega ; A):=\left\{g \in \mathcal{H}^{1}(\Omega): A g \in L_{2}(\rho ; \Omega)\right\}, \quad\|g\|_{\mathcal{H}^{1,0}(\Omega ; A)}^{2}:=\|g\|_{\mathcal{H}^{1}(\Omega)}^{2}+\|\rho A g\|_{L_{2}(\Omega)}^{2}$.

If $u \in \mathcal{H}^{1,0}(\Omega ; A)$, one can correctly define the canonical co-normal derivative $T^{+} u \in$ $H^{-\frac{1}{2}}(\partial \Omega)$ similar to, for example, [5, Lemma 3.2], [14, Lemma 4.3]) as

$$
\left\langle T^{+} u, w\right\rangle_{\partial \Omega}:=\int_{\Omega}\left[\left(\gamma_{-1}^{+} w\right) A u+E\left(u, \gamma_{-1}^{+} w\right)\right] d x \quad \forall w \in H^{\frac{1}{2}}(\partial \Omega),
$$

where $\gamma_{-1}^{+}: H^{\frac{1}{2}}(\partial \Omega) \rightarrow \mathcal{H}^{1}(\Omega)$ is a bounded right inverse to the trace operator $\gamma^{+}: \mathcal{H}^{1}(\Omega) \rightarrow H^{\frac{1}{2}}(\partial \Omega)$. The symbol $\left\langle g_{1}, g_{2}\right\rangle_{\partial \Omega}$ denotes the duality brackets between the spaces $H^{-\frac{1}{2}}(\partial \Omega)$ and $H^{\frac{1}{2}}(\partial \Omega)$, coinciding with $\int_{\partial \Omega} g_{1}(x) g_{2}(x) d S$ if $g_{1}, g_{2} \in L_{2}(\partial \Omega)$. The operator $T^{+}: \mathcal{H}^{1,0}(\Omega ; A) \rightarrow H^{-\frac{1}{2}}(\partial \Omega)$ is continuous and gives the continuous extension on $\mathcal{H}^{1,0}(\Omega ; A)$ of the classical co-normal derivative operator $a \partial_{n}$, where $\partial_{n}=n \cdot \nabla$ and $n=n^{+}$is normal vector on $\partial \Omega$ directed outward the exterior domain $\Omega$. When $a \equiv 1$, we employ for $T^{+}$the notation $T_{\Delta}^{+}$, which is the continuous extension on $\mathcal{H}^{1,0}(\Omega ; \Delta)$ of the classical normal derivative operator $\partial_{n}$.

Similar to the proofs available in [5, Lemma 3.4], [14, Lemma 4.3]) (see also [18] for the spaces $H^{s, t}(\Omega ; A)$ ), one can prove that for $u \in \mathcal{H}^{1,0}(\Omega ; A)$ the first Green identity holds in the form

$$
\left\langle T^{+} u, \gamma^{+} v\right\rangle_{\partial \Omega}=\int_{\Omega}[v A u+E(u, v)] d x \quad \forall v \in \mathcal{H}^{1}(\Omega) .
$$

Then for any functions $u, v \in \mathcal{H}^{1,0}(\Omega ; A)$ we have the second Green identity,

$$
\int_{\Omega}[v A u-u A v] d x=\left\langle T^{+} u, \gamma^{+} v\right\rangle_{\partial \Omega}-\left\langle T^{+} v, \gamma^{+} u\right\rangle_{\partial \Omega} .
$$

\section{Boundary Value Problems}

The mixed boundary value problem in an exterior domain $\Omega$ is defined as follows.

Find a function $u \in \mathcal{H}^{1,0}(\Omega ; A)$ satisfying the conditions

$$
\begin{aligned}
& A u=f \quad \text { in } \quad \Omega, \\
& \gamma^{+} u=\varphi_{0} \quad \text { on } \partial_{D} \Omega, \\
& T^{+} u=\psi_{0} \quad \text { on } \quad \partial_{N} \Omega
\end{aligned}
$$

where

$$
\varphi_{0} \in H^{\frac{1}{2}}\left(\partial_{D} \Omega\right), \quad \psi_{0} \in H^{-\frac{1}{2}}\left(\partial_{N} \Omega\right), \quad f \in L_{2}(\rho ; \Omega) .
$$

Here $\partial \Omega=\overline{\partial_{D} \Omega} \cup \overline{\partial_{N} \Omega}$, while $\partial_{D} \Omega \neq \varnothing$ and $\partial_{N} \Omega \neq \varnothing$ are nonintersecting simply connected sub-manifolds of $\partial \Omega$ with an infinitely smooth boundary curve $\ell:=\overline{\partial_{D} \Omega} \cap$ $\overline{\partial_{N} \Omega} \in C^{\infty}$.

If $\partial_{N} \Omega=\varnothing$, i.e. $\partial_{D} \Omega=\partial \Omega$, then we arrive at the Dirichlet problem for $u \in \mathcal{H}^{1,0}(\Omega ; A)$,

$$
\begin{aligned}
& A u=f \quad \text { in } \quad \Omega, \\
& \gamma^{+} u=\varphi_{0} \quad \text { on } \partial \Omega,
\end{aligned}
$$


where $\varphi_{0} \in H^{\frac{1}{2}}(\partial \Omega), \quad f \in L_{2}(\rho ; \Omega)$.

If $\partial_{D} \Omega=\varnothing$, i.e. $\partial_{N} \Omega=\partial \Omega$ in (3.1)-(3.4), then we arrive at the Neumann problem for $u \in \mathcal{H}^{1,0}(\Omega ; A)$,

$$
\begin{aligned}
& A u=f \text { in } \Omega, \\
& T^{+} u=\psi_{0} \text { on } \partial \Omega,
\end{aligned}
$$

where $\psi_{0} \in H^{-\frac{1}{2}}(\partial \Omega), \quad f \in L_{2}(\rho ; \Omega)$.

Let us denote by

$$
\begin{aligned}
& \mathcal{A}_{M}: \mathcal{H}^{1,0}(\Omega ; A) \rightarrow L_{2}(\rho ; \Omega) \times H^{\frac{1}{2}}\left(\partial_{D} \Omega\right) \times H^{-\frac{1}{2}}\left(\partial_{N} \Omega\right) \\
& \mathcal{A}_{D}: \mathcal{H}^{1,0}(\Omega ; A) \rightarrow L_{2}(\rho ; \Omega) \times H^{\frac{1}{2}}(\partial \Omega) \\
& \mathcal{A}_{N}: \mathcal{H}^{1,0}(\Omega ; A) \rightarrow L_{2}(\rho ; \Omega) \times H^{-\frac{1}{2}}(\partial \Omega)
\end{aligned}
$$

the left hand side operators of, respectively, the mixed BVP (3.1)-(3.3), the Dirichlet BVP (3.5)-(3.6) and the Neumann BVP (3.7)-(3.8), which are evidently continuous. The following assertion follows from Theorems 8.1, 8.3 and 8.6 proved in Appendix using variational settings and the Lax-Milgram lemma. It is similar to the results of $[24,11]$ for a general divergent form elliptic equation in $\mathbb{R}^{n}$ and of $[8,13,7]$ for the Dirichlet and Neumann problems for the Poisson equation in an exterior domain $\Omega$ with a compact boundary.

Theorem 3.1. Under conditions (2.6) the mixed, Dirichlet and Neumann homogeneous problems are uniquely solvable in $\mathcal{H}^{1,0}(\Omega ; A)$ and the corresponding inverse operators

$$
\begin{aligned}
& \mathcal{A}_{M}^{-1}: L_{2}(\rho ; \Omega) \times H^{\frac{1}{2}}\left(\partial_{D} \Omega\right) \times H^{-\frac{1}{2}}\left(\partial_{N} \Omega\right) \rightarrow \mathcal{H}^{1,0}(\Omega ; A), \\
& \mathcal{A}_{D}^{-1}: L_{2}(\rho ; \Omega) \times H^{\frac{1}{2}}(\partial \Omega) \rightarrow \mathcal{H}^{1,0}(\Omega ; A), \\
& \mathcal{A}_{N}^{-1}: L_{2}(\rho ; \Omega) \times H^{-\frac{1}{2}}(\partial \Omega) \rightarrow \mathcal{H}^{1,0}(\Omega ; A)
\end{aligned}
$$

are continuous.

\section{Parametrix and Parametrix-Based Potentials}

It is well known, cf. $[15,2]$, that the function

$$
P(x, y)=\frac{-1}{4 \pi a(y)|x-y|}, \quad x, y \in \mathbb{R}^{3},
$$

is a parametrix (Levi function) for the operator $A\left(x, \partial_{x}\right)$, i.e.,

$$
A\left(x, \partial_{x}\right) P(x, y)=\delta(x-y)+R(x, y)
$$

where

$$
R(x, y)=\sum_{i=1}^{3} \frac{x_{i}-y_{i}}{4 \pi a(y)|x-y|^{3}} \frac{\partial a(x)}{\partial x_{i}}, \quad x, y \in \mathbb{R}^{3} .
$$

The parametrix $P(x, y)$ is related to a fundamental solution to the operator $A\left(y, \partial_{x}\right):=$ $a(y) \Delta_{x}$ with the "frozen" coefficient $a(x)=a(y)$, and $A\left(y, \partial_{x}\right) P(x, y)=\delta(x-y)$.

To obtain boundary-domain integral equations, we will consider the coefficient $a$ such that

$$
a \in C^{1}\left(\mathbb{R}^{3}\right) \text { and } \rho \nabla a \in L_{\infty}\left(\mathbb{R}^{3}\right) .
$$


Remark 4.1. One can check that if $a$ satisfies (2.6) and the second condition in (4.4), then $\|g a\|_{\mathcal{H}^{1}(\Omega)} \leq C_{1}\|g\|_{\mathcal{H}^{1}(\Omega)},\|g / a\|_{\mathcal{H}^{1}(\Omega)} \leq C_{2}\|g\|_{\mathcal{H}^{1}(\Omega)}$, where the constants $C_{1}$ and $C_{2}$ are independent of $g \in \mathcal{H}^{1}(\Omega)$, i.e., $a$ and $1 / a$ are multipliers in the space $\mathcal{H}^{1}(\Omega)$.

For any fixed $y \in \Omega$ and any ball $B_{\epsilon}(y)$ centered at $y$ with sufficiently small radius $\epsilon>0$, we have $R(., y) \in L_{2}\left(\rho ; \Omega \backslash B_{\epsilon}(y)\right)$ and thus $P(., y) \in \mathcal{H}^{1,0}\left(\Omega \backslash B_{\epsilon}(y)\right)$ by $(4.2)$. Applying the second Green identity (2.9) in $\Omega \backslash B_{\epsilon}(y)$ with $v=P(y, \cdot)$ and taking usual limits as $\epsilon \rightarrow 0$, cf. [21], we get the third Green identity,

$$
u+\mathcal{R} u-V\left(T^{+} u\right)+W\left(\gamma^{+} u\right)=\mathcal{P} A u \quad \text { in } \Omega
$$

for any $u \in \mathcal{H}^{1,0}(\Omega ; A)$. Here

$$
\mathcal{P} g(y):=\int_{\Omega} P(x, y) g(x) d x, \quad \mathcal{R} g(y):=\int_{\Omega} R(x, y) g(x) d x, \quad y \in \mathbb{R}^{3},
$$

are, respectively, the parametrix-based volume Newton-type and remainder potentials, while

$$
\begin{array}{r}
V g(y):=-\int_{\partial \Omega} P(x, y) g(x) d S_{x}, \quad W g(y):=-\int_{\partial \Omega}\left[T_{x} P(x, y)\right] g(x) d S_{x}, \\
y \in \mathbb{R}^{3} \backslash \partial \Omega,
\end{array}
$$

are the parametrix-based surface single layer and double layer potentials. The Newtontype and the remainder potential operators given by (4.6) for $\Omega=\mathbb{R}^{3}$ will be denoted as $\mathbf{P}$ and $\mathbf{R}$, respectively. Recall that in the definition of $W$ we assumed $T_{x}=a(x) n(x) \cdot \nabla_{x}$, where $n=n^{+}$is normal vector on $\partial \Omega$ directed outward the exterior domain $\Omega$. Note that if the integrands in (4.6), (4.7) and further on in the paper do not belong to $L_{1}$, then the integrals should be understood as the corresponding duality forms (or limits of these forms for the infinitely smooth functions, existing due to the function density in the corresponding Sobolev spaces).

From definitions (4.1), (4.3), (4.6)-(4.7) one can obtain representations of the parametrix-based potential operators in terms of their counterparts for $a=1$ (i.e. associated with the Laplace operator $\Delta$ ), which we equip with the subscript $\Delta$, cf. [2],

$$
\begin{aligned}
\mathcal{P} g & =\frac{1}{a} \mathcal{P}_{\Delta} g, & \mathcal{R} g & =-\frac{1}{a} \sum_{j=1}^{3} \partial_{j}\left[\mathcal{P}_{\Delta}\left(g \partial_{j} a\right)\right], \\
V g & =\frac{1}{a} V_{\Delta} g, & W g & =\frac{1}{a} W_{\Delta}(a g) .
\end{aligned}
$$

In addition to conditions (2.6), (4.4) on the coefficient $a$, we will sometimes also need the condition

$$
\rho^{2} \Delta a \in L_{\infty}\left(\mathbb{R}^{3}\right) .
$$

Theorem 4.1. The following operators are continuous under the second condition in $(4.4)$,

$$
\begin{aligned}
\mathbf{P} & : \mathcal{H}^{-1}\left(\mathbb{R}^{3}\right) \rightarrow \mathcal{H}^{1}\left(\mathbb{R}^{3}\right), \\
\mathcal{P} & : \widetilde{\mathcal{H}}^{-1}(\Omega) \rightarrow \mathcal{H}^{1}\left(\mathbb{R}^{3}\right), \\
\mathbf{R} & : L_{2}\left(\rho^{-1} ; \mathbb{R}^{3}\right) \rightarrow \mathcal{H}^{1}\left(\mathbb{R}^{3}\right), \\
V & : H^{-\frac{1}{2}}(\partial \Omega) \rightarrow \mathcal{H}^{1}(\Omega), \\
W & : H^{\frac{1}{2}}(\partial \Omega) \rightarrow \mathcal{H}^{1}(\Omega),
\end{aligned}
$$


while the following operators are continuous under the second condition in (4.4) and condition (4.10),

$$
\begin{aligned}
\mathcal{P} & : L_{2}(\rho ; \Omega) \rightarrow \mathcal{H}^{1,0}\left(\mathbb{R}^{3} ; A\right), \\
\mathcal{R} & : \mathcal{H}^{1}(\Omega) \rightarrow \mathcal{H}^{1,0}(\Omega ; A), \\
V & : H^{-\frac{1}{2}}(\partial \Omega) \rightarrow \mathcal{H}^{1,0}(\Omega ; A), \\
W & : H^{\frac{1}{2}}(\partial \Omega) \rightarrow \mathcal{H}^{1,0}(\Omega ; A) .
\end{aligned}
$$

Proof. Let $\phi \in \mathcal{D}\left(\mathbb{R}^{3}\right) \subset \mathcal{H}^{-1}\left(\mathbb{R}^{3}\right)$. Then the Newton potential

$$
\mathbf{P}_{\Delta} \phi=\frac{-1}{4 \pi} \int_{\mathbb{R}^{3}} \frac{\phi(x)}{|x-y|} d x
$$

evidently belongs to $\mathcal{H}^{1}\left(\mathbb{R}^{3}\right)$ and solves the Poisson equation $\Delta v=\phi$ in $\mathbb{R}^{3}$. On the other hand, the Laplace operator from $\mathcal{H}^{1}\left(\mathbb{R}^{3}\right)$ to $\mathcal{H}^{-1}\left(\mathbb{R}^{3}\right)$ possesses a continuous inverse operator $\Delta^{-1}: \mathcal{H}^{-1}\left(\mathbb{R}^{3}\right) \rightarrow \mathcal{H}^{1}\left(\mathbb{R}^{3}\right)$, see e.g. [24, Theorem 1.2], [11, Theorem III.2]. Thus $\mathbf{P}_{\Delta} \phi=\Delta^{-1} \phi$, which due to the density of $\mathcal{D}\left(\mathbb{R}^{3}\right)$ in $\mathcal{H}^{-1}\left(\mathbb{R}^{3}\right)$ gives a continuous extension of $\mathbf{P}_{\Delta}$ to the operator $\mathcal{H}^{-1}\left(\mathbb{R}^{3}\right) \rightarrow \mathcal{H}^{1}\left(\mathbb{R}^{3}\right)$. Then the first relation in (4.8) implies (4.11) under condition (4.4), and thus (4.12) immediately follows.

To prove (4.16), let us denote by $\tilde{g}$ the extension of a function $g \in L_{2}(\rho ; \Omega)$ by zero outside $\Omega$. Evidently $\tilde{g} \in L_{2}\left(\rho ; \mathbb{R}^{3}\right) \subset \mathcal{H}^{-1}\left(\mathbb{R}^{3}\right)$ and $\mathcal{P}_{\Delta} g=\mathbf{P}_{\Delta} \tilde{g} \in \mathcal{H}^{1}\left(\mathbb{R}^{3}\right)$. Taking into account that

$$
A \mathcal{P} g=g-\sum_{j=1}^{3} \partial_{j}\left(\frac{\partial_{j} a}{a} \mathcal{P}_{\Delta} g\right),
$$

conditions (4.4) and (4.10) imply (4.16).

Let us prove the continuity of operators (4.14) and (4.18). For $\phi \in \mathcal{C}^{\infty}(\partial \Omega)$ let us consider the single layer potential for the Laplace operator,

$$
V_{\Delta} \phi=\frac{1}{4 \pi} \int_{\partial \Omega} \frac{1}{|x-y|} \phi(x) d \Gamma(x)
$$

which evidently belongs to $\mathcal{H}^{1}(\Omega ; \Delta)$ and solves the Dirichlet problem

$$
\Delta v=0 \text { in } \Omega, \quad \gamma^{+} v=w \text { on } \partial \Omega
$$

for $v \in \mathcal{H}^{1}(\Omega ; \Delta)$, where $w=\gamma V_{\Delta} \phi$. By Theorem 3.1, problem (4.20) is uniquely solvable and its solution is delivered by a continuous operator $Q: H^{\frac{1}{2}}(\partial \Omega) \rightarrow \mathcal{H}^{1,0}(\Omega ; \Delta)$, i.e., $V_{\Delta} \phi=Q \gamma V_{\Delta} \phi$. Taking into account the continuity of the operator $\gamma V_{\Delta}: H^{-\frac{1}{2}}(\partial \Omega) \rightarrow$ $H^{\frac{1}{2}}(\partial \Omega)$ and the density of $C^{\infty}(\partial \Omega)$ in $H^{-\frac{1}{2}}(\partial \Omega)$, we arrive at the continuity of $V_{\Delta}$ : $H^{-\frac{1}{2}}(\partial \Omega) \rightarrow \mathcal{H}^{1}(\Omega ; \Delta)$. Then the first relation in (4.9) implies continuity of (4.14) under conditions (4.4) and of (4.18) under conditions (4.4), (4.10). Continuity of (4.15) and (4.19) is proved by a similar argument.

To prove continuity of (4.13), let us consider $g \in L_{2}\left(\rho^{-1} ; \mathbb{R}^{3}\right)$. Since the operator of multiplication with $\partial_{j} a$ is continuous from $L_{2}\left(\rho^{-1} ; \mathbb{R}^{3}\right)$ to $L_{2}\left(\mathbb{R}^{3}\right)$ due to conditions (4.4), 
we have $g \partial_{j} a \in L_{2}\left(\mathbb{R}^{3}\right)$. The second relation in (4.8) gives

$$
\begin{aligned}
\mathbf{R} g(y) & =\frac{1}{4 \pi a(y)} \sum_{j=1}^{3} \int_{\mathbb{R}^{3}}\left[\partial_{y_{j}} \frac{1}{|x-y|}\right] g(x) \partial_{j} a(x) d x \\
& =-\frac{1}{4 \pi a(y)} \sum_{j=1}^{3} \int_{\mathbb{R}^{3}}\left[\partial_{x_{j}} \frac{1}{|x-y|}\right] g(x) \partial_{j} a(x) d x \\
& =\frac{1}{4 \pi a(y)} \sum_{j=1}^{3} \int_{\mathbb{R}^{3}} \frac{1}{|x-y|} \partial_{j}\left(g(x) \partial_{j} a(x)\right) d x=-\sum_{j=1}^{3} \mathbf{P}\left[\partial_{j}\left(g \partial_{j} a\right)\right](y) .
\end{aligned}
$$

To justify the Gauss divergence theorem employed in (4.21), one can introduce a sequence of functions from $\mathcal{D}\left(\mathbb{R}^{3}\right)$ converging to $g \partial_{j} a$ in $L_{2}\left(\mathbb{R}^{3}\right)$, which gradients will then converge to the gradient of $g \partial_{j} a$ in $H^{-1}\left(\mathbb{R}^{3}\right)$ and thus in $\mathcal{H}^{-1}\left(\mathbb{R}^{3}\right)$. Then continuity of (4.11) implies continuity of (4.13).

Let us prove continuity of (4.17). Since $\mathcal{H}^{1}(\Omega)$ is continuously embedded in $\subset$ $L_{2}\left(\rho^{-1} ; \Omega\right)$, then the continuity of the operator $\mathcal{R}: \mathcal{H}^{1}(\Omega) \rightarrow \mathcal{H}^{1}(\Omega)$ is implied by (4.13). For any $g \in \mathcal{H}^{1}(\Omega)$ we have,

$$
\begin{aligned}
A \mathcal{R} g & =\sum_{k}^{3} \partial_{k}\left(a \partial_{k} \mathcal{R}\right)=\nabla a \cdot \nabla \mathcal{R} g+a \Delta \mathcal{R} g \\
& =\nabla a \cdot \nabla \mathcal{R} g+a^{2}\left[\Delta\left(\frac{1}{a}\right)\right] \mathcal{R} g+2 a \nabla\left(\frac{1}{a}\right) \cdot \nabla(a \mathcal{R} g)+\Delta(a \mathcal{R} g) .
\end{aligned}
$$

By the second relation in (4.8),

$$
\Delta(a \mathcal{R} g)=-\sum_{j=1}^{3} \partial_{j} \Delta \mathcal{P}_{\Delta}\left(g \partial_{j} a\right)=-\nabla g \cdot \nabla a-g \Delta a .
$$

Then (4.22) along with conditions (4.4) and (4.10) imply continuity of the operator $A \mathcal{R}$ : $\mathcal{H}^{1}(\Omega) \rightarrow L_{2}(\rho ; \Omega)$ and thus of the operator (4.17).

Let us introduce the following boundary integral (pseudodifferential) operators of the direct values and of the co-normal derivatives of the single and double layer potentials:

$$
\begin{aligned}
& \mathcal{V} g(y):=-\int_{S} P(x, y) g(x) d S_{x}, \\
& \mathcal{W} g(y):=-\int_{S}\left[T\left(x, n(x), \partial_{x}\right) P(x, y)\right] g(x) d S_{x}, \\
& \mathcal{W}^{\prime} g(y):=-\int_{S}\left[T\left(y, n(y), \partial_{y}\right) P(x, y)\right] g(x) d S_{x}, \\
& \mathcal{L}^{ \pm} g(y):=T^{ \pm} W g(y),
\end{aligned}
$$

where $y \in S$. 
They can be also presented in terms of their counterparts for $a=1$, i.e. associated with the Laplace operator $\Delta$, cf. [2],

$$
\begin{aligned}
& \mathcal{V} g=\frac{1}{a} \mathcal{V}_{\Delta} g, \quad \mathcal{W} g=\frac{1}{a} \mathcal{W}_{\Delta}(a g) \\
& \mathcal{W}^{\prime} g=\mathcal{W}_{\Delta}^{\prime} g+\left[a \frac{\partial}{\partial n}\left(\frac{1}{a}\right)\right] \mathcal{V}_{\Delta} g \\
& \mathcal{L}^{ \pm} g=\mathcal{L}_{\Delta}(a g)+\left[a \frac{\partial}{\partial n}\left(\frac{1}{a}\right)\right] W_{\Delta}^{ \pm}(a g)
\end{aligned}
$$

where, as usual, the subscript $\Delta$ means that the corresponding surface potentials are based on the harmonic fundamental solution $P_{\Delta}(x, y)=-(4 \pi|x-y|)^{-1}$. It is taken into account that $a$ and its first derivatives are continuous in $\mathbb{R}^{3}$ and

$$
\hat{\mathcal{L}} g:=\mathcal{L}_{\Delta}(a g):=\mathcal{L}_{\Delta}^{+}(a g)=\mathcal{L}_{\Delta}^{-}(a g)
$$

by the Lyapunov-Tauber theorem.

The mapping and jump properties of the operators (4.23)-(4.26) follow from relations (4.27)-(4.29) and are described in details in [2]. Particularly, their jump relations are given by the following theorem presented in [2, Theorem 3.3].

Theorem 4.2. Let $g_{1} \in H^{-\frac{1}{2}}(S)$, and $g_{2} \in H^{\frac{1}{2}}(S)$ and $a \in C^{1}\left(\mathbb{R}^{3}\right)$. Then

$$
\begin{aligned}
& \gamma^{ \pm} V g_{1}(y)=\mathcal{V} g_{1}(y) \\
& \gamma^{ \pm} W g_{2}(y)=\mp \frac{1}{2} g_{2}(y)+\mathcal{W} g_{2}(y), \\
& T^{ \pm} V g_{1}(y)= \pm \frac{1}{2} g_{1}(y)+\mathcal{W}^{\prime} g_{1}(y),
\end{aligned}
$$

where $y \in \partial \Omega$.

Taking trace and co-normal derivative of the third Green identity (4.5), we obtain,

$$
\begin{aligned}
& \frac{1}{2} \gamma^{+} u+\gamma^{+} \mathcal{R} u-\mathcal{V} T^{+} u+\mathcal{W} \gamma^{+} u=\gamma^{+} \mathcal{P} A u \quad \text { on } \partial \Omega, \\
& \frac{1}{2} T^{+} u+T^{+} \mathcal{R} u-\mathcal{W}_{\partial \Omega}^{\prime} T^{+} u+\mathcal{L}_{\partial \Omega}^{+} \gamma^{+} u=T^{+} \mathcal{P} A u \quad \text { on } \partial \Omega .
\end{aligned}
$$

For arbitrary functions $u, f, \Psi, \Phi$, let us consider a more general "indirect" integral relation, associated with (4.5),

$$
u+\mathcal{R} u-V \Psi+W \Phi=\mathcal{P} f \quad \text { in } \Omega,
$$

and prove for the weighted spaces the analog of [2, Lemma 4.1].

Lemma 4.1. Let $u \in \mathcal{H}^{1}(\Omega), f \in L_{2}(\rho ; \Omega), \Psi \in H^{-\frac{1}{2}}(\partial \Omega), \Phi \in H^{\frac{1}{2}}(\partial \Omega)$ satisfy $(4.33)$ and conditions (4.4), (4.10) hold. Then $u$ belongs to $\mathcal{H}^{1,0}(\Omega ; A)$ and is a solution of the equation

$$
A u=f \quad \text { in } \quad \Omega,
$$

while

$$
V\left(\Psi-T^{+} u\right)-W(\Phi-\gamma+u)=0 \text { in } \Omega .
$$

Proof. First of all, rewriting (4.33) in the form $u=\mathcal{P} f-\mathcal{R} u+V \Psi-W \Phi$, we conclude by Theorem 4.1 that $u \in \mathcal{H}^{1,0}(\Omega ; A)$. Thus we can write the third Green identity (4.5) for the function $u$. 
Subtracting (4.33) from the identity (4.5), we obtain

$$
-V \Psi^{*}+W \Phi^{*}=\mathcal{P}[A u-f] \quad \text { in } \Omega,
$$

where $\Psi^{*}:=T^{+} u-\Psi, \Phi^{*}:=\gamma^{+} u-\Phi$. Multiplying equality (4.36) by $a(y)$ we get

$$
-V_{\Delta} \Psi^{*}+W_{\Delta}\left(a \Phi^{*}\right)=\mathcal{P}_{\Delta}[A u-f] \text { in } \Omega .
$$

Applying the Laplace operator $\Delta$ to the last equation and taking into consideration that the both functions in the left-hand side are harmonic surface potentials, while the right-hand side function is the classical Newtonian volume potential, we arrive at equation (4.34). Substituting (4.34) back into (4.36) leads to (4.35). form.

The counterpart of $[2$, Lemma 4.2] for an unbounded domain $\Omega$ takes the following

Lemma 4.2. Let conditions (4.4), (4.10) hold.

(i) If $\Psi^{*} \in H^{-\frac{1}{2}}(\partial \Omega)$ and $V \Psi^{*}=0$ in $\Omega$, then $\Psi^{*}=0$.

(ii) If $\Phi^{*} \in H^{\frac{1}{2}}(\partial \Omega)$ and $W \Phi^{*}(y)=0$ in $\Omega$, then $\Phi^{*}(x)=C / a(x)$, where $C$ is a constant.

(iii) Let $\partial \Omega=\overline{S_{1}} \cup \overline{S_{2}}$, where $S_{1}$ and $S_{2}$ are nonempty nonintersecting simply connected submanifolds of $\partial \Omega$ with infinitely smooth boundaries. If $\Psi^{*} \in \widetilde{H}^{-\frac{1}{2}}\left(S_{1}\right), \Phi^{*} \in \widetilde{H}^{\frac{1}{2}}\left(S_{2}\right)$ and $V \Psi^{*}(y)-W \Phi^{*}(y)=0$ in $\Omega$, then $\Psi^{*}=0$ and $\Phi^{*}=0$ on $\partial \Omega$.

Proof. The proofs of items (i) and (iii) coincide with the proofs of their counterparts for interior domains in [2, Lemma 4.2].

To prove item (ii), we first remark that $\Phi_{\Delta}=C$ satisfies the equation $W_{\Delta} \Phi_{\Delta}=0$ in the exterior domain $\Omega$ for any $C=$ const. (This follows from the first Green identity (2.8) for the interior domain $\Omega^{-}$employed for $v(x)=C, A=\Delta, u=-1 /(4 \pi|x-y|)$ and for any $y \in \Omega$.) Let us check that there is no other solution of the equation in $\Omega$ in $H^{\frac{1}{2}}(\partial \Omega)$. By the Lyapunov-Tauber theorem, $T_{\Delta}^{+} W_{\Delta} \Phi_{\Delta}=T_{\Delta}^{-} W_{\Delta} \Phi_{\Delta}=0$ on $\partial \Omega$, which implies $W_{\Delta} \Phi_{\Delta}=$ const in the interior domain $\Omega^{-}$due to the uniqueness up to a constant of the solution of the Neumann problem in $H^{1}\left(\Omega^{-}\right)$. Then the jump property of the double layer potential gives $\Phi_{\Delta}=$ const. Applying the second relation of (4.9) finalizes the proof of item (ii).

\section{Segregated BDIEs for the Mixed Problem}

Let us fix an extension $\Phi_{0} \in H^{\frac{1}{2}}(S)$ of the given function $\varphi_{0}$ in the Dirichlet boundary condition (3.2) from $\partial_{D} \Omega$ to the whole of $\partial \Omega$ and an extension $\Psi_{0} \in H^{-\frac{1}{2}}(S)$ of the given function $\psi_{0}$ in the Neumann boundary condition (3.3) from $\partial_{N} \Omega$ to the whole of $\partial \Omega$.

We will explore different possibilities of reducing BVP (3.1)-(3.3) to a system of Boundary-Domain Integral Equations (BDIEs) and in all of them we represent in (4.5), (4.31) and (4.32) the trace of the function $u$ and in its co-normal derivative as

$$
\gamma^{+} u=\Phi_{0}+\varphi, \quad \varphi \in \widetilde{H}^{\frac{1}{2}}\left(\partial_{N} \Omega\right) ; \quad T^{+} u=\Psi_{0}+\psi, \quad \psi \in \widetilde{H}^{-\frac{1}{2}}\left(\partial_{D} \Omega\right),
$$

and will regard the new unknown functions $\varphi$ and $\psi$ as formally segregated of $u$. Thus we will look for the triplet

$$
\begin{array}{r}
\mathcal{U}=(u, \psi, \varphi)^{\top} \in \mathbb{H}:=\mathcal{H}^{1,0}(\Omega ; A) \times \widetilde{H}^{-\frac{1}{2}}\left(\partial_{D} \Omega\right) \times \widetilde{H}^{\frac{1}{2}}\left(\partial_{N} \Omega\right) \\
\subset \mathbb{X}:=\mathcal{H}^{1}(\Omega) \times \widetilde{H}^{-\frac{1}{2}}\left(\partial_{D} \Omega\right) \times \widetilde{H}^{\frac{1}{2}}\left(\partial_{N} \Omega\right) .
\end{array}
$$


BDIE system (M11). First, using equation (4.5) in $\Omega$, the restriction of equation (4.31) on $\partial_{D} \Omega$, and the restriction of equation (4.32) on $\partial_{N} \Omega$, we arrive at the BDIE system (M11) of three equations for the triplet of unknowns, $(u, \psi, \varphi)$,

$$
\begin{array}{rlrl}
u+\mathcal{R} u-V \psi+W \varphi & =F_{0} & \text { in } \quad \Omega, \\
r_{\partial_{D} \Omega}\left\{\gamma^{+} \mathcal{R} u-\mathcal{V} \psi+\mathcal{W} \varphi\right\} & =r_{\partial_{D} \Omega} \gamma^{+} F_{0}-\varphi_{0} & & \text { on } \quad \partial_{D} \Omega, \\
r_{\partial_{N} \Omega}\left\{T^{+} \mathcal{R} u-\mathcal{W}^{\prime} \psi+\mathcal{L}^{+} \varphi\right\} & =r_{\partial_{N} \Omega} T^{+} F_{0}-\psi_{0} & & \text { on } \quad \partial_{N} \Omega,
\end{array}
$$

where

$$
F_{0}:=\mathcal{P} f+V \Psi_{0}-W \Phi_{0} \quad \text { in } \quad \Omega
$$

We denote the matrix operator of the left hand side of the systems (M11) as

$$
\mathcal{M}^{11}:=\left[\begin{array}{ccc}
I+\mathcal{R} & -V & W \\
r_{\partial_{D} \Omega} \gamma^{+} \mathcal{R} & -r_{\partial_{D} \Omega} \mathcal{V} & r_{\partial_{D} \Omega} \mathcal{W} \\
r_{\partial_{N} \Omega} T^{+} \mathcal{R} & -r_{\partial_{N} \Omega} \mathcal{W}^{\prime} & r_{\partial_{N} \Omega} \mathcal{L}^{+}
\end{array}\right]
$$

The notation (M11) and the corresponding superscripts mean that the system includes the integral operators of the first kind both on the Dirichlet and Neumann parts of the boundary. The other BDIE systems below are also denoted respectively.

BDIE system (M12). If we use equation (4.5) in $\Omega$ and equation (4.31) on the whole of $\partial \Omega$, we arrive at the BDIE system (M12) of two equations for the triplet $(u, \psi, \varphi)$,

$$
\begin{aligned}
& u+\mathcal{R} u-V \psi+W \varphi=F_{0} \quad \text { in } \Omega, \\
& \frac{1}{2} \varphi+\gamma^{+} \mathcal{R} u-\mathcal{V} \psi+\mathcal{W} \varphi=\gamma^{+} F_{0}-\Phi_{0} \quad \text { on } \quad \partial \Omega
\end{aligned}
$$

The left hand side matrix operator of the system is

$$
\mathcal{M}^{12}:=\left[\begin{array}{ccc}
I+\mathcal{R} & -V & W \\
\gamma^{+} \mathcal{R} & -\mathcal{V} & \frac{1}{2} I+\mathcal{W}
\end{array}\right]
$$

BDIE system (M21). Using equation (4.5) in $\Omega$ and equation (4.32) on the whole of $\partial \Omega$, we arrive at the BDIE system (M21) of two equations for the triplet $(u, \psi, \varphi)$,

$$
\begin{aligned}
& u+\mathcal{R} u-V \psi+W \varphi=F_{0} \quad \text { in } \Omega, \\
& \frac{1}{2} \psi+T^{+} \mathcal{R} u-\mathcal{W}^{\prime} \psi+\mathcal{L}^{+} \varphi=T^{+} F_{0}-\Psi_{0} \quad \text { on } \quad \partial \Omega \text {. }
\end{aligned}
$$

The left hand side matrix operator of the system is

$$
\mathcal{M}^{21}:=\left[\begin{array}{ccc}
I+\mathcal{R} & -V & W \\
T^{+} \mathcal{R} & \frac{1}{2} I-\mathcal{W}^{\prime} & \mathcal{L}^{+}
\end{array}\right] .
$$


BDIE system (M22). Finally, using equation (4.5) in $\Omega$, the restriction of equation (4.32) on $\partial_{D} \Omega$, and the restriction of equation (4.31) on $\partial_{N} \Omega$, we arrive for the triplet $(u, \psi, \varphi)$ at the BDIE system (M22) of three equations of "almost" the second kind (up to the spaces),

$$
\begin{aligned}
u+\mathcal{R} u-V \psi+W \varphi & =F_{0} & & \text { in } \quad \Omega, \\
\frac{1}{2} \psi+r_{\partial_{D} \Omega}\left\{T^{+} \mathcal{R} u-\mathcal{W}^{\prime} \psi+\mathcal{L}^{+} \varphi\right\} & =r_{\partial_{D} \Omega}\left\{T^{+} F_{0}-\Psi_{0}\right\} & & \text { on } \quad \partial_{D} \Omega, \\
\frac{1}{2} \varphi+r_{\partial_{N} \Omega}\left\{\gamma^{+} \mathcal{R} u-\mathcal{V} \psi+\mathcal{W} \varphi\right\} & =r_{\partial_{N} \Omega}\left\{\gamma^{+} F_{0}-\Phi_{0}\right\} & & \text { on } \quad \partial_{N} \Omega .
\end{aligned}
$$

The matrix operator of the left hand side of the system (M22) takes form

$$
\mathcal{M}^{22}:=\left[\begin{array}{ccc}
I+\mathcal{R} & -V & W \\
r_{\partial_{D} \Omega} T^{+} \mathcal{R} & r_{\partial_{D} \Omega}\left(\frac{1}{2} I-\mathcal{W}^{\prime}\right) & r_{\partial_{D} \Omega} \mathcal{L}^{+} \\
r_{\partial_{N} \Omega} \gamma^{+} \mathcal{R} & -r_{\partial_{N} \Omega} \mathcal{V} & r_{\partial_{N} \Omega}\left(\frac{1}{2} I+\mathcal{W}\right)
\end{array}\right]
$$

Remark 5.1. Note that the second relation in (4.8) means that if $a=$ const outside a bounded subdomain $\Omega^{\prime} \subset \Omega$, then the operator $\mathcal{R}$ acts only on the restriction $r_{\Omega^{\prime}} u$. This implies that all the BDIE systems reduce in this case to the BDIEs over $\Omega^{\prime}$ and $\partial \Omega$, that are supplemented with the integral representations for $u$ in $\Omega \backslash \bar{\Omega}^{\prime}$ given by the first equations of the systems.

The systems (M11), (M12), (M21) and (M22) can be rewritten as

$$
\mathcal{M}^{\alpha \beta} \mathcal{U}=\mathcal{F}^{\alpha \beta}
$$

where $\mathcal{F}^{\alpha \beta}$ denote their right hand sides and $\alpha, \beta=1,2$. If conditions (4.4) and (4.10) hold, then due to the mapping properties of the potentials, $\mathcal{F}^{\alpha \beta} \in \mathbb{F}^{\alpha \beta} \subset \mathbb{Y}^{\alpha \beta}$, while the operators $\mathcal{M}^{\alpha \beta}: \mathbb{H} \rightarrow \mathbb{F}^{\alpha \beta}$ and $\mathcal{M}^{\alpha \beta}: \mathbb{X} \rightarrow \mathbb{Y}^{\alpha \beta}$ are continuous for any $\alpha, \beta=1,2$. Here we denoted

$$
\begin{aligned}
\mathbb{F}^{11}: & =\mathcal{H}^{1,0}(\Omega ; A) \times H^{\frac{1}{2}}\left(\partial_{D} \Omega\right) \times H^{-\frac{1}{2}}\left(\partial_{N} \Omega\right), \\
\mathbb{F}^{12}: & =\mathcal{H}^{1,0}(\Omega ; A) \times H^{\frac{1}{2}}(\partial \Omega), \\
\mathbb{F}^{21}: & =\mathcal{H}^{1,0}(\Omega ; A) \times H^{-\frac{1}{2}}(\partial \Omega), \\
\mathbb{F}^{22}: & =\mathcal{H}^{1,0}(\Omega ; A) \times H^{-\frac{1}{2}}\left(\partial_{D} \Omega\right) \times H^{\frac{1}{2}}\left(\partial_{N} \Omega\right), \\
\mathbb{Y}^{11}: & =\mathcal{H}^{1}(\Omega) \times H^{\frac{1}{2}}\left(\partial_{D} \Omega\right) \times H^{-\frac{1}{2}}\left(\partial_{N} \Omega\right), \\
\mathbb{Y}^{12}: & =\mathcal{H}^{1}(\Omega) \times H^{\frac{1}{2}}(\partial \Omega), \\
\mathbb{Y}^{21}: & =\mathcal{H}^{1}(\Omega) \times H^{-\frac{1}{2}}(\partial \Omega), \\
\mathbb{Y}^{22}: & =\mathcal{H}^{1}(\Omega) \times H^{-\frac{1}{2}}\left(\partial_{D} \Omega\right) \times H^{\frac{1}{2}}\left(\partial_{N} \Omega\right) .
\end{aligned}
$$

\section{Equivalence and Uniqueness Theorems}

Let us first prove the equivalence theorems.

Theorem 6.1. Let $\varphi_{0} \in H^{\frac{1}{2}}\left(\partial_{D} \Omega\right), \psi_{0} \in H^{-\frac{1}{2}}\left(\partial_{N} \Omega\right), f \in L_{2}(\rho ; \Omega)$ and let $\Phi_{0} \in H^{\frac{1}{2}}(\partial \Omega)$ and $\Psi_{0} \in H^{-\frac{1}{2}}(\partial \Omega)$ be some extensions of $\varphi_{0}$ and $\psi_{0}$, respectively, and conditions (4.4), (4.10) hold. 
(i) If a function $u \in \mathcal{H}^{1,0}(\Omega ; A)$ solves the $B V P(3.1)-(3.3)$, then the triplet $(u, \psi, \varphi)$, where

$$
\psi=T^{+} u-\Psi_{0} \in \widetilde{H}^{-\frac{1}{2}}\left(\partial_{D} \Omega\right), \quad \varphi=\gamma^{+} u-\Phi_{0} \in \widetilde{H}^{\frac{1}{2}}\left(\partial_{N} \Omega\right),
$$

solves the BDIE systems (M11), (M12), (M21) and (M22).

(ii) If a triplet $(u, \psi, \varphi) \in \mathcal{H}^{1,0}(\Omega ; A) \times \widetilde{H}^{-\frac{1}{2}}\left(\partial_{D} \Omega\right) \times \widetilde{H}^{\frac{1}{2}}\left(\partial_{N} \Omega\right)$ solves one of the BDIE systems (M11), (M12) or (M22), then this solution is unique and solves all the systems, including (M21), while u solves BVP (3.1)-(3.3) and relations (6.1) hold.

Proof. Item (i) immediately follows from the deduction of the BDIE systems (M11), (M12), (M21) and (M22).

Using the similarity of Lemma 4.1 and items (i, iii) of Lemma 4.2 to their counterparts, Lemma 4.1 and Lemma 4.2(i, iii) in [2], for the bounded domain $\Omega$, the proof of item (ii) of the theorem follows word-for-word the corresponding proofs of Theorems 5.2, 5.6 and 5.12 in $[2]$.

The situation with uniqueness and equivalence for system (M21) differs from the one for other systems and from its counterpart BDIE system $(\mathcal{T} \mathcal{T})$ in [2], particularly because item (ii) of Lemma 4.2 is different from its analog, Lemma 4.2(ii) in [2]. This leads to the following assertion.

Theorem 6.2. Let $\varphi_{0} \in H^{\frac{1}{2}}\left(\partial_{D} \Omega\right), \psi_{0} \in H^{-\frac{1}{2}}\left(\partial_{N} \Omega\right), f \in L_{2}(\rho ; \Omega)$ and let $\Phi_{0} \in H^{\frac{1}{2}}(\partial \Omega)$ and $\Psi_{0} \in H^{-\frac{1}{2}}(\partial \Omega)$ be some extensions of $\varphi_{0}$ and $\psi_{0}$, respectively, and conditions (4.4), (4.10) hold.

(i) Homogeneous BDIE system (M21) admits only one linearly independent solution $\left(u^{0}, \psi^{0}, \varphi^{0}\right) \in \mathcal{H}^{1,0}(\Omega ; A) \times \widetilde{H}^{-\frac{1}{2}}\left(\partial_{D} \Omega\right) \times \widetilde{H}^{\frac{1}{2}}\left(\partial_{N} \Omega\right)$, where $u^{0}$ is the solution of the mixed $B V P$

$$
\begin{aligned}
A u^{0} & =0 \quad \text { in } \quad \Omega, \\
r_{\partial_{D} \Omega} \gamma^{+} u^{0} & =\frac{1}{a(x)} \quad \text { on } \quad \partial_{D} \Omega, \\
r_{\partial_{N} \Omega} T^{+} u^{0} & =0 \quad \text { on } \quad \partial_{N} \Omega,
\end{aligned}
$$

while

$$
\psi^{0}=T^{+} u^{0}, \quad \varphi^{0}=\gamma^{+} u^{0}-1 / a(x) \quad \text { on } \quad \partial \Omega .
$$

(ii) The non-homogeneous BDIE systems (M21) is solvable, and any its solution $(u, \psi, \varphi) \in \mathcal{H}^{1,0}(\Omega ; A) \times \widetilde{H}^{-\frac{1}{2}}\left(\partial_{D} \Omega\right) \times \widetilde{H}^{\frac{1}{2}}\left(\partial_{N} \Omega\right)$ can be represented as

$$
u=\tilde{u}+C u^{0} \text { in } \Omega,
$$

where $\tilde{u}$ solves the BVP (3.1)-(3.3) and $C$ is a constant, while

$$
\psi=T^{+} \tilde{u}-\Psi_{0}+C \psi^{0}, \quad \varphi=\gamma^{+} \tilde{u}-\Phi_{0}+C \varphi^{0} \quad \text { on } \quad \partial \Omega .
$$

Proof. Problem (6.2)-(6.4) is uniquely solvable in $\mathcal{H}^{1,0}(\Omega ; A)$ by Theorem 3.1. Consequently, the third Green identity (4.5) is applicable to $u^{0}$, leading to

$$
u^{0}+\mathcal{R} u^{0}-V \psi^{0}+W \varphi^{0}=0 \quad \text { in } \Omega,
$$

with notations (6.5), if we take into account that $W(1 / a(x))=0$ in $\Omega$ due to the second relation in (4.9) and the equality $W_{\Delta} 1=0$ in $\Omega$ (cf. the proof of Lemma 4.2(ii)). Taking 
the co-normal derivative of (6.8) and substituting the first equation of (6.5) again, we arrive at

$$
\frac{1}{2} \psi^{0}+T^{+} \mathcal{R} u^{0}-\mathcal{W}^{\prime} \psi^{0}+\mathcal{L}^{+} \varphi^{0}=0 \quad \text { on } \quad \partial \Omega .
$$

Equations (6.8)-(6.9) mean that the triplet $\left(u^{0}, \psi^{0}, \varphi^{0}\right)$ solves the homogeneous BDIE system (M21).

To prove item (ii) and check that there exists only one linearly independent solution of the homogeneous BDIE system (M21), we proceed as follows. First, we remark that the solvability of the non-homogeneous system (M21) follows from the solvability of the BVP (3.1)-(3.3) in $\mathcal{H}^{1,0}(\Omega ; A)$ and the deduction of system (M21).

Let now a triplet $(u, \psi, \varphi)^{\top} \in \mathcal{H}^{1,0}(\Omega ; A) \times \widetilde{H}^{-\frac{1}{2}}\left(\partial_{D} \Omega\right) \times \widetilde{H}^{\frac{1}{2}}\left(\partial_{N} \Omega\right)$ solve (generally non-homogeneous) BDIE system (M21). Take the co-normal derivative of equation (5.2) on $\partial \Omega$ and subtract it from equation (5.3) to obtain

$$
\psi+\Psi_{0}-T^{+} u=0 \quad \text { on } \quad \partial \Omega .
$$

Taking into account that $\psi=0$ on $\partial_{N} \Omega$ and $\Psi_{0}=\psi_{0}$ on $\partial_{N} \Omega$, this implies that $u$ satisfies the Neumann condition (3.3).

Equations (5.2) and (5.1) and Lemma 4.1 with $\Psi=\psi+\Psi_{0}, \Phi=\varphi+\Phi_{0}$ imply that $u$ is a solution of equation (3.1) and

$$
V\left(\Psi_{0}+\psi-T^{+} u\right)-W\left(\Phi_{0}+\varphi-\gamma^{+} u\right)=0 \quad \text { in } \quad \Omega .
$$

Due to (6.10) the first term vanishes in (6.11), and by Lemma 4.2(ii) we obtain

$$
\Phi_{0}+\varphi-\gamma^{+} u=-C / a(x) \text { on } \partial \Omega
$$

where $C$ is a constant. Taking into account that $\varphi=0$ on $\partial_{D} \Omega$ and $\Phi_{0}=\varphi_{0}$ on $\partial_{D} \Omega$, we conclude that $u$ satisfies the Dirichlet condition

$$
\gamma^{+} u=\varphi_{0}+C / a(x) \text { on } \partial_{D} \Omega
$$

instead of (3.2). Introducing notation $\tilde{u}$ by (6.6) in (6.10), (6.12) and (6.13) and taking into account (6.2)-(6.4) prove the claim of item (ii).

The case $\varphi_{0}=0, \Phi_{0}=0, \psi_{0}=0, \Psi_{0}=0, f=0$ leading to the homogeneous BDIE system (M21) also implies that $\tilde{u}$ for this case satisfies homogeneous BVP (3.1)-(3.3) and thus $\tilde{u}=0$ in $(6.6)$ and $(6.7)$ meaning that the triplet $\left(u^{0}, \psi^{0}, \varphi^{0}\right)$ is the only linearly independent solution of the homogeneous BDIE system (M21). This completes the proof of item (i) and of the whole theorem.

\section{BDIO Fredholm Properties and Invertibility}

We will consider in this section the Fredholm properties and invertibility of the boundarydomain integral operators (BDIOs), starting from $\mathcal{M}^{\alpha \beta}: \mathbb{H} \rightarrow \mathbb{F}^{\alpha \beta}$ and then, under more restrictive conditions on the coefficient $a$, of the operators $\mathcal{M}^{\alpha \beta}: \mathbb{X} \rightarrow \mathbb{Y}^{\alpha \beta}, \alpha, \beta=1,2$.

\subsection{Properties of operators $\mathcal{M}^{\alpha \beta}: \mathbb{H} \rightarrow \mathbb{F}^{\alpha \beta}$}

In this section, we will analyze the operator invertibility (or the Fredholm property when there is no invertibility) by proving first that the arbitrary right hand side functions from the corresponding spaces can be represented in terms of the parametrix-based potentials and using then the equivalence theorems. 
To start with, let us prove the following analog of Lemma 5.5 in [17] for the exterior domain.

Lemma 7.1. For any function $\mathcal{F}_{*} \in \mathcal{H}^{1,0}(\Omega ; A)$, there exists a unique couple $\left(f_{*}, \Psi_{*}\right)=$ $\mathcal{C F}_{*} \in L_{2}(\rho ; \Omega) \times H^{-\frac{1}{2}}(\partial \Omega)$ such that

$$
\mathcal{F}_{*}(y)=\mathcal{P} f_{*}(y)+V \Psi_{*}(y), \quad y \in \Omega,
$$

where $\mathcal{C}: \mathcal{H}^{1,0}(\Omega ; A) \rightarrow L_{2}(\rho ; \Omega) \times H^{-\frac{1}{2}}(\partial \Omega)$ is a linear bounded operator.

Proof. Suppose first that there exist some functions $f_{*}(y)$ and $\Psi_{*}(y)$ satisfying (7.1) and find their expressions in terms of $\mathcal{F}_{*}(y)$. Taking into account relations (4.8) and (4.9) for the volume and single layer potentials, ansatz (7.1) can be rewritten as

$$
a(y) \mathcal{F}_{*}(y)=\mathcal{P}_{\Delta} f_{*}(y)+V_{\Delta} \Psi_{*}(y), \quad y \in \Omega .
$$

Applying the Laplace operator to (7.2) we obtain that

$$
f_{*}=\Delta\left(a \mathcal{F}_{*}\right) \text { in } \Omega .
$$

Then (7.2) can be rewritten as

$$
V_{\Delta} \Psi_{*}(y)=Q(y), \quad y \in \Omega,
$$

where

$$
Q(y):=a(y) \mathcal{F}_{*}(y)-\mathcal{P}_{\Delta}\left[\Delta\left(a \mathcal{F}_{*}\right)\right](y), \quad y \in \Omega .
$$

The trace of (7.4) on the boundary gives

$$
\mathcal{V}_{\Delta} \Psi_{*}(y)=\gamma^{+} Q(y), \quad y \in \partial \Omega,
$$

where $\mathcal{V}_{\Delta}:=\left.\mathcal{V}\right|_{a=1}$ is the direct value on $\partial \Omega$ of the single layer operator $V_{\Delta}$ associated with the Laplace operator. Since $\mathcal{V}_{\Delta}: H^{s}(\partial \Omega) \rightarrow H^{s+1}(\partial \Omega), s \in \mathbb{R}$, is isomorphism (c.f. e.g. [6, Ch. XI, Part B, $\S 2$, Remark 1]), we obtain the following expression for $\Psi_{*}$

$$
\Psi_{*}(y)=\mathcal{V}_{\Delta}^{-1} \gamma^{+} Q(y), \quad y \in \partial \Omega .
$$

Relations (7.3) and (7.7) imply uniqueness of the couple $f_{*}, \Psi_{*}$. Now we have to prove that $f_{*}(y), \Psi_{*}(y)$ given by (7.3) and (7.7) satisfy (7.1). Indeed, the potential $V_{\Delta} \Psi_{*}(y)$ with $\Psi_{*}(y)$ given by (7.7) is a harmonic function, and one can check that $Q$ given by (7.5) is also harmonic in $\Omega$. Then condition (7.6) implies that $V_{\Delta} \Psi_{*}(y)$ and $Q(y)$ coincide in the $\Omega$ (cf. Theorem 3.1), i.e. (7.4) holds true, which implies (7.1). Thus (7.3), (7.7) and (7.5) give

$$
\left(f_{*}, \Psi_{*}\right)=\mathcal{C} \mathcal{F}_{*}:=\left(\Delta\left(a F_{*}\right), \mathcal{V}_{\Delta}^{-1} \gamma^{+}\left[a F_{*}-\mathcal{P}_{\Delta} \Delta\left(a F_{*}\right)\right]\right),
$$

and thus by Remark 4.1, $\mathcal{C}: \mathcal{H}^{1,0}(\Omega ; A) \rightarrow L_{2}(\rho ; \Omega) \times H^{-\frac{1}{2}}(\partial \Omega)$ is a bounded operator $\square$

Corollary 7.1. A couple $\left(\mathcal{F}_{0}, \mathcal{F}_{1}\right) \in \mathcal{H}^{1,0}(\Omega ; A) \times H^{\frac{1}{2}}(\partial \Omega)$ can be uniquely represented as

$$
\begin{aligned}
& \mathcal{F}_{0}=\mathcal{P} f_{*}+V \Psi_{*}-W \Phi_{*} \quad \text { in } \quad \Omega, \\
& \mathcal{F}_{1}=\gamma^{+} \mathcal{F}_{0}-\Phi_{*} \quad \text { on } \quad \partial \Omega
\end{aligned}
$$

for some $\left(f_{*}, \Psi_{*}, \Phi_{*}\right)=\mathcal{C}_{*}\left(\mathcal{F}_{0}, \mathcal{F}_{1}\right)^{\top}$, where $\mathcal{C}_{*}: \mathcal{H}^{1,0}(\Omega ; A) \times H^{\frac{1}{2}}(\partial \Omega) \rightarrow L_{2}(\rho ; \Omega) \times$ $H^{-\frac{1}{2}}(\partial \Omega) \times H^{\frac{1}{2}}(\partial \Omega)$ is a linear bounded operator. 
Proof. Taking $\Phi_{*}=\gamma^{+} \mathcal{F}_{0}-\mathcal{F}_{1}$ and applying Lemma 7.1 to $\mathcal{F}_{*}=\mathcal{F}_{0}+W \Phi_{*}$, we prove existence of representation (7.8)-(7.9). To prove its uniqueness, we consider its homogeneous case, i.e., with $\mathcal{F}_{0}=0, \mathcal{F}_{1}=0$. Then (7.9) implies $\Phi_{*}=0$ and thus by (7.8) and Lemma 7.1 we also obtain $\Psi_{*}=0, f_{*}=0$.

Using essentially the same reasoning as in Lemma 7.1 and Corollary 7.1 , one can prove the following statement, that is similar to its counterpart for bounded domains, see $[2$, Lemma 5.13 and Corollary 5.14].

Lemma 7.2. Let $\partial \Omega=\overline{S_{1}} \cup \overline{S_{2}}$, where $S_{1}$ and $S_{2}$ are nonempty nonintersecting simply connected submanifolds of $\partial \Omega$ with infinitely smooth boundaries. For an arbitrary triplet

$$
\mathcal{F}=\left(\mathcal{F}_{0}, \mathcal{F}_{1}, \mathcal{F}_{2}\right)^{\top} \in \mathcal{H}^{1,0}(\Omega ; A) \times H^{-\frac{1}{2}}\left(S_{1}\right) \times H^{\frac{1}{2}}\left(S_{2}\right)
$$

there exists a unique triplet

$$
\left(f_{*}, \Psi_{*}, \Phi_{*}\right)^{\top}=\mathcal{C}_{S_{1}, S_{2}} \mathcal{F} \in L_{2}(\rho ; \Omega) \times H^{-\frac{1}{2}}(\partial \Omega) \times H^{\frac{1}{2}}(\partial \Omega)
$$

such that

$$
\begin{aligned}
& \mathcal{F}_{0}=\mathcal{P} f_{*}+V \Psi_{*}-W \Phi_{*} \text { in } \Omega^{+}, \\
& \mathcal{F}_{1}=r_{S_{1}} T^{+} \mathcal{F}_{0}-r_{S_{1}} \Psi_{*} \text { on } S_{1}, \\
& \mathcal{F}_{2}=r_{S_{2}} \gamma^{+} \mathcal{F}_{0}-r_{S_{2}} \Phi_{*} \text { on } S_{2},
\end{aligned}
$$

where $\mathcal{C}_{S_{1}, S_{2}}: \mathcal{H}^{1,0}(\Omega ; A) \times H^{-\frac{1}{2}}\left(S_{1}\right) \times H^{\frac{1}{2}}\left(S_{2}\right) \rightarrow L_{2}(\rho ; \Omega) \times H^{-\frac{1}{2}}(\partial \Omega) \times H^{\frac{1}{2}}(\partial \Omega)$ is a linear bounded operator.

Theorem 7.1. If conditions (4.4) and (4.10) hold, then the operators

$$
\mathcal{M}^{11}: \mathbb{H} \rightarrow \mathbb{F}^{11}, \quad \mathcal{M}^{12}: \mathbb{H} \rightarrow \mathbb{F}^{12}, \quad \mathcal{M}^{22}: \mathbb{H} \rightarrow \mathbb{F}^{22}
$$

are continuous and continuously invertible.

Proof. Continuity of operators (7.14) follows from the volume and boundary potential mapping properties, Theorem 4.1 .

Let us prove continuous invertibility of the operator $\mathcal{M}^{11}: \mathbb{H} \rightarrow \mathbb{F}^{11}$. By Lemma 7.2, any right hand side $\mathcal{F}^{11}=\left(\mathcal{F}_{0}, \mathcal{F}_{D}, \mathcal{F}_{N}\right) \in \mathbb{F}^{11}$ of the equation $\mathcal{M}^{11} \mathcal{U}=\mathcal{F}^{11}$ can be uniquely represented in form (7.11)-(7.13) with $S_{1}=\partial_{N} \Omega, S_{2}=\partial_{D} \Omega$, where $\left(f_{*}, \Psi_{*}, \Phi_{*}\right)^{\top}=\mathcal{C}_{\partial_{D} \Omega, \partial_{N} \Omega} \mathcal{F}^{11}$ and the operator $\mathcal{C}_{\partial_{N} \Omega, \partial_{D} \Omega}: \mathbb{F}^{11}=\mathcal{H}^{1,0}(\Omega ; A) \times$ $H^{\frac{1}{2}}\left(\partial_{D} \Omega\right) \times H^{-\frac{1}{2}}\left(\partial_{N} \Omega\right) \rightarrow L_{2}(\rho ; \Omega) \times H^{-\frac{1}{2}}(\partial \Omega) \times H^{\frac{1}{2}}(\partial \Omega)$ is continuous.

Then equivalence Theorem 6.1 for the system (M11) and invertibility Theorem 3.1 for the mixed problem imply that the equation $\mathcal{M}^{11} \mathcal{U}=\mathcal{F}^{11}$ has a solution $\mathcal{U}=(u, \psi, \varphi)^{\top}=$ $\left(\mathcal{M}^{11}\right)^{-1} \mathcal{F}^{11}$, where the operator $\left(\mathcal{M}^{11}\right)^{-1}: \mathbb{F}^{11} \rightarrow \mathbb{H}$ is given by

$$
u=\mathcal{A}_{M}^{-1}\left[f_{*}, r_{\partial_{D} \Omega} \Phi_{*}, r_{\partial_{N} \Omega} \Psi_{*}\right]^{\top}, \quad \psi=T^{+} u-\Psi_{*}, \quad \varphi=\gamma^{+} u-\Phi_{*},
$$

where $\left(f_{*}, \Psi_{*}, \Phi_{*}\right)^{\top}=\mathcal{C}_{\partial_{D} \Omega, \partial_{N} \Omega} \mathcal{F}^{11}$, and is evidently continuous. Thus the operator $\left(\mathcal{M}^{11}\right)^{-1}$ is the right inverse to the operator $\mathcal{M}^{11}: \mathbb{H} \rightarrow \mathbb{F}^{11}$, but due to the injectivity of the latter implied by the equivalence Theorem 6.1 , the operator $\left(\mathcal{M}^{11}\right)^{-1}$ is in fact the two-side inverse.

Continuous invertibility of the operator $\mathcal{M}^{22}: \mathbb{H} \rightarrow \mathbb{F}^{22}$ is proved similarly. 
Let us prove continuous invertibility of the operator $\mathcal{M}^{12}: \mathbb{H} \rightarrow \mathbb{F}^{12}$. By Corollary 7.1, any right hand side $\mathcal{F}^{12}=\left(\mathcal{F}_{0}, \mathcal{F}_{1}\right) \in \mathbb{F}^{12}$ of the equation $\mathcal{M}^{12} \mathcal{U}=\mathcal{F}^{12}$ can be uniquely represented in form (7.8)-(7.9) for some $\left(f_{*}, \Psi_{*}, \Phi_{*}\right)^{\top}=\mathcal{C}_{*} \mathcal{F}^{12}$, where the operator $\mathcal{C}_{*}$ : $\mathbb{F}^{12}=\mathcal{H}^{1,0}(\Omega ; A) \times H^{\frac{1}{2}}(\partial \Omega) \rightarrow L_{2}(\rho ; \Omega) \times H^{-\frac{1}{2}}(S) \times H^{\frac{1}{2}}(S)$ is continuous.

Then equivalence Theorem 6.1 for the system (M12) and invertibility Theorem 3.1 for the mixed problem imply that the equation $\mathcal{M}^{12} \mathcal{U}=\mathcal{F}^{12}$ has a solution $\mathcal{U}=(u, \psi, \varphi)^{\top}=$ $\left(\mathcal{M}^{12}\right)^{-1} \mathcal{F}^{12}$, where the operator $\left(\mathcal{M}^{12}\right)^{-1}: \mathbb{F}^{12} \rightarrow \mathbb{H}$ is given by expressions (7.15), where $\left(f_{*}, \Psi_{*}, \Phi_{*}\right)^{\top}=\mathcal{C}_{*} \mathcal{F}^{12}$, and is evidently continuous. Thus the operator $\left(\mathcal{M}^{12}\right)^{-1}$ is the right inverse to the operator $\mathcal{M}^{12}: \mathbb{H} \rightarrow \mathbb{F}^{12}$, but due to the injectivity of the latter implied by the equivalence Theorem 6.1 , the operator $\left(\mathcal{M}^{12}\right)^{-1}$ is in fact the two-side inverse.

Let us prove an assertion implied by Theorem 7.1 for the operator $\mathcal{M}^{22}: \mathbb{H} \rightarrow \mathbb{F}^{22}$ for the particular case $a=1$ in $\Omega$, i.e., essentially for the purely boundary integral equation. We will need it to prove invertibility of the operator $\mathcal{M}^{22}: \mathbb{X} \rightarrow \mathbb{Y}^{22}$ for variable $a$ in Section 7.2.

If $a=1$ in $\Omega$, then (3.1) becomes the classical Laplace equation, the remainder operator $\mathcal{R}$ vanishes, and the BDIE system (M22) splits into the system of two Boundary Integral Equations (BIEs),

$$
\begin{gathered}
r_{\partial_{D} \Omega}\left(\frac{1}{2} \psi-\mathcal{W}_{\Delta}^{\prime} \psi+\mathcal{L}_{\Delta}^{+} \varphi\right)=r_{\partial_{D} \Omega} T^{+} F_{0}-r_{\partial_{D} \Omega} \Psi_{0} \text { on } \partial_{D} \Omega \\
r_{\partial_{N} \Omega}\left(\frac{1}{2} \varphi-\mathcal{V}_{\Delta} \psi+\mathcal{W}_{\Delta} \varphi\right)=r_{\partial_{N} \Omega} F_{0}^{+}-r_{\partial_{N} \Omega} \Phi_{0} \text { on } \partial_{N} \Omega
\end{gathered}
$$

and the representation formula for $u$ in terms of $\varphi$ and $\psi$,

$$
u=F_{0}+V_{\Delta} \psi-W_{\Delta} \varphi \text { in } \Omega .
$$

System (7.16)-(7.17) can be rewritten in the form

$$
\hat{\mathcal{M}}_{\Delta}^{22} \hat{\mathcal{U}}_{\Delta}=\hat{\mathcal{F}}_{\Delta}^{22}
$$

where $\hat{\mathcal{U}}_{\Delta}^{\top}:=(\psi, \varphi) \in \widetilde{H}^{-\frac{1}{2}}\left(\partial_{D} \Omega\right) \times \widetilde{H}^{\frac{1}{2}}\left(\partial_{N} \Omega\right)$,

$$
\begin{aligned}
\hat{\mathcal{M}}_{\Delta}^{22} & :=\left[\begin{array}{cc}
r_{\partial_{D} \Omega}\left(\frac{1}{2} I-\mathcal{W}_{\Delta}^{\prime}\right) & r_{\partial_{D} \Omega} \mathcal{L}_{\Delta}^{+} \\
-r_{\partial_{N} \Omega} \mathcal{V}_{\Delta} & r_{\partial_{N} \Omega}\left(\frac{1}{2} I+\mathcal{W}_{\Delta}\right)
\end{array}\right], \\
\hat{\mathcal{F}}_{\Delta}^{22} & :=\left[\begin{array}{c}
r_{\partial_{D} \Omega} T^{+} F_{0}-r_{\partial_{D} \Omega} \Psi_{0} \\
r_{\partial_{N} \Omega} F_{0}^{+}-r_{\partial_{N} \Omega} \Phi_{0}
\end{array}\right] \in H^{-\frac{1}{2}}\left(\partial_{D} \Omega\right) \times H^{\frac{1}{2}}\left(\partial_{N} \Omega\right) .
\end{aligned}
$$

Moreover, the operator $\hat{\mathcal{M}}_{\Delta}^{22}: \widetilde{H}^{-\frac{1}{2}}\left(\partial_{D} \Omega\right) \times \widetilde{H}^{\frac{1}{2}}\left(\partial_{N} \Omega\right) \rightarrow H^{-\frac{1}{2}}\left(\partial_{D} \Omega\right) \times H^{\frac{1}{2}}\left(\partial_{N} \Omega\right)$ is bounded and by Theorem 6.1 (employed for $a=1$ ) is also injective.

Theorem 7.2. The operator $\hat{\mathcal{M}}_{\Delta}^{22}: \widetilde{H}^{-\frac{1}{2}}\left(\partial_{D} \Omega\right) \times \widetilde{H}^{\frac{1}{2}}\left(\partial_{N} \Omega\right) \rightarrow H^{-\frac{1}{2}}\left(\partial_{D} \Omega\right) \times H^{\frac{1}{2}}\left(\partial_{N} \Omega\right)$ is continuously invertible.

Proof. A solution of system (7.18) with an arbitrary $\left(\hat{\mathcal{F}}_{\Delta}^{22}\right)^{\top}=\left(\mathcal{F}_{1 \Delta}^{22}, \mathcal{F}_{2 \Delta}^{22}\right) \in$ $H^{-\frac{1}{2}}\left(\partial_{D} \Omega\right) \times H^{\frac{1}{2}}\left(\partial_{N} \Omega\right)$ is delivered by the couple $(\psi, \varphi)$ satisfying the extended system 


$$
\begin{aligned}
\mathcal{M}_{\Delta}^{22} \mathcal{U}=\mathcal{F}_{\Delta}^{0}, \text { where } \mathcal{U} & =(u, \psi, \varphi)^{\top}, \mathcal{F}_{\Delta}^{0}=\left(0, \mathcal{F}_{1 \Delta}^{22}, \mathcal{F}_{2 \Delta}^{22}\right)^{\top}, \text { and } \\
\mathcal{M}_{\Delta}^{22} & :=\left[\begin{array}{ccc}
I & -V_{\Delta} & W_{\Delta} \\
0 & r_{\partial_{D} \Omega}\left(\frac{1}{2} I-\mathcal{W}_{\Delta}^{\prime}\right) & r_{\partial_{D} \Omega} \mathcal{L}_{\Delta}^{+} \\
0 & -r_{\partial_{N} \Omega} \mathcal{V}_{\Delta} & r_{\partial_{N} \Omega}\left(\frac{1}{2} I+\mathcal{W}_{\Delta}\right)
\end{array}\right] .
\end{aligned}
$$

The operator $\mathcal{M}_{\Delta}^{22}: \mathbb{H} \rightarrow \mathbb{F}^{22}$ has a continuous inverse due to Theorem 7.1 for $a=1$. Consequently, the operator $\hat{\mathcal{M}}_{\Delta}^{22}$ has a right bounded inverse, which is also a two-side inverse due to injectivity of the operator $\hat{\mathcal{M}}_{\Delta}^{22}$.

To analyze properties of the operator $\mathcal{M}^{21}$, we will need the following assertion, that appeared to be quite different from its counterpart for interior domains proved in $[16$, Lemma 19].

Lemma 7.3. If conditions (4.4) and (4.10) hold, then a function $\mathcal{F}_{*} \in \mathcal{H}^{1,0}(\Omega ; A)$ can be represented as

$$
\mathcal{F}_{*}(y)=\mathcal{P} f_{*}(y)-W \Phi_{*}(y), \quad y \in \Omega,
$$

for some $\left(f_{*}, \Phi_{*}\right) \in L_{2}(\rho ; \Omega) \times H^{\frac{1}{2}}(\partial \Omega)$ if and only if

$$
\int_{\partial \Omega} T_{\Delta}^{+}\left(a \mathcal{F}_{*}\right) d S=0
$$

Proof. Suppose first there exist some functions $f_{*}(y)$ and $\Phi_{*}(y)$ satisfying (7.20). Taking into account relations (4.8) and (4.9) for the Newton-type and double layer potentials, ansatz (7.20) can be rewritten as

$$
a(y) \mathcal{F}_{*}(y)=\mathcal{P}_{\Delta} f_{*}(y)-W_{\Delta}\left[a \Phi_{*}\right](y), \quad y \in \Omega .
$$

Applying the Laplace operator to (7.22) we obtain that

$$
f_{*}=\Delta\left(a \mathcal{F}_{*}\right) \text { in } \Omega .
$$

Then (7.22) can be rewritten as

$$
W_{\Delta}\left[a \Phi_{*}\right](y)=Q(y), \quad y \in \Omega
$$

where

$$
Q(y):=\mathcal{P}_{\Delta}\left[\Delta\left(a \mathcal{F}_{*}\right)\right](y)-a(y) \mathcal{F}_{*}(y), \quad y \in \Omega .
$$

The trace of (7.24) on the boundary gives

$$
\left[-\frac{1}{2} I+\mathcal{W}_{\Delta}\right]\left(a \Phi_{*}\right)=\gamma^{+} Q, \quad \text { on } \quad \partial \Omega
$$

By [6, Ch. XI, Part B, $\S 2$, Theorem 4]), equation (7.26) admits a solution $a \Phi_{*} \in H^{\frac{1}{2}}(\partial \Omega)$ if and only if the right hand side $\gamma^{+} Q \in H^{\frac{1}{2}}(\partial \Omega)$ satisfies the condition

$$
\int_{\partial \Omega} \gamma^{+} Q(x) T_{\Delta}^{+} v(x) d S_{x}=0
$$

where $v \in \mathcal{H}^{1}(\Omega)$ solves the Dirichlet problem $\Delta v=0$ in $\Omega, \gamma^{+} v=1$ on $\partial \Omega$. Employing the second Green identity (2.9) associated with the operator $\Delta$ and substituting there (7.25), we have

$$
\int_{\partial \Omega} T_{\Delta}^{+}\left\{\mathcal{P}_{\Delta}\left[\Delta\left(a \mathcal{F}_{*}\right)\right]-a \mathcal{F}_{*}\right\} d S=0
$$


We have $T_{\Delta}^{+} \mathcal{P}_{\Delta}\left[\Delta\left(a \mathcal{F}_{*}\right)\right]=T_{\Delta}^{-} \mathcal{P}_{\Delta}\left[\Delta\left(a \mathcal{F}_{*}\right)\right]$ on $\partial \Omega$ since $\mathcal{P}_{\Delta}\left[\Delta\left(a \mathcal{F}_{*}\right)\right] \in \mathcal{H}^{1,0}\left(\mathbb{R}^{3} ; \Delta\right) \subset$ $H_{\text {loc }}^{2}\left(\mathbb{R}^{3}\right)$ by Theorem 4.1. Keeping in mind that $\mathcal{P}_{\Delta}\left[\Delta\left(a \mathcal{F}_{*}\right)\right]$ is a harmonic function in the bounded domain $\Omega^{-}$, we obtain

$$
\int_{\partial \Omega} T_{\Delta}^{+} \mathcal{P}_{\Delta}\left[\Delta\left(a \mathcal{F}_{*}\right)\right] d S=0
$$

which reduces $(7.28)$ to $(7.21)$.

Let now (7.21) be satisfied. We have to prove that there exist a representation (7.20). First of all, let us note that if $\mathcal{F}_{*} \in \mathcal{H}^{1,0}(\Omega ; A)$, then conditions (4.4) and (4.10) imply $a \mathcal{F}_{*} \in \mathcal{H}^{1,0}(\Omega ; \Delta)$ and the co-normal derivative $T_{\Delta}^{+}\left(a \mathcal{F}_{*}\right)$ is well defined on $\partial \Omega$. Then (7.21) implies (7.28). Let $a \Phi_{*} \in H^{\frac{1}{2}}(\partial \Omega)$ be a solution of (7.26) with $Q$ given by (7.25), while $f_{*} \in L_{2}(\rho ; \Omega)$ be given by (7.23). Then the potential $W_{\Delta}\left[a \Phi_{*}\right] \in \mathcal{H}^{1}(\Omega)$ is a harmonic function, and one can check that $Q \in \mathcal{H}^{1}(\Omega)$ is also harmonic. Since (7.26) implies that they coincide on the boundary, the two harmonic functions should coincide also in the domain, cf. Theorem 3.1, i.e. (7.24) holds true, which implies (7.20).

Lemma 7.3 implies the following corollary.

Corollary 7.2. If conditions (4.4) and (4.10) hold, then a couple $\left(\mathcal{F}_{0}, \mathcal{F}_{1}\right) \in \mathcal{H}^{1,0}(\Omega ; A) \times$ $H^{-\frac{1}{2}}(\partial \Omega)$ can be represented as

$$
\begin{aligned}
& \mathcal{F}_{0}(y)=\mathcal{P} f_{*}(y)+V \Psi_{*}(y)-W \Phi_{*}(y), \quad y \in \Omega, \\
& \mathcal{F}_{1}(y)=T^{+} \mathcal{F}_{0}(y)-\Psi_{*}(y), \quad y \in \partial \Omega
\end{aligned}
$$

for some $\left(f_{*}, \Psi_{*}, \Phi_{*}\right) \in L_{2}(\rho ; \Omega) \times H^{-\frac{1}{2}}(\partial \Omega) \times H^{\frac{1}{2}}(\partial \Omega)$ if and only if

$$
\left.\left.g^{0}\left(\mathcal{F}_{0}, \mathcal{F}_{1}\right):=\int_{\partial \Omega}\left[\left(\partial_{n} a\right) \gamma^{+} \mathcal{F}_{0}+\mathcal{F}_{1}\right)\right]\right\} d S=0 .
$$

Proof. We take $\Psi_{*}=T^{+} \mathcal{F}_{0}-\mathcal{F}_{1}$ and apply Lemma 7.3 to $\mathcal{F}_{*}=\mathcal{F}_{0}-V \Psi_{*}$, which proves representation (7.29) if and only if

$$
\int_{\partial \Omega} T_{\Delta}^{+}\left[a\left(\mathcal{F}_{0}-V\left(T^{+} \mathcal{F}_{0}-\mathcal{F}_{1}\right)\right)\right] d S=0
$$

Taking into account the jump property of the single layer potential and that $a V g \equiv V_{\Delta} g$ is a harmonic function in the bounded domain $\Omega^{-}$, condition (7.32) reduces to

$$
\begin{aligned}
0 & =\int_{\partial \Omega}\left[\left(T_{\Delta}^{+} a\right) \gamma^{+} \mathcal{F}_{0}+a T_{\Delta}^{+} \mathcal{F}_{0}-T^{+} \mathcal{F}_{0}+\mathcal{F}_{1}\right] d S-\int_{\partial \Omega} T_{\Delta}^{-} V_{\Delta}\left(T^{+} \mathcal{F}_{0}-\mathcal{F}_{1}\right) d S \\
& \left.\left.=\int_{\partial \Omega}\left[\left(\partial_{n} a\right) \gamma^{+} \mathcal{F}_{0}+\mathcal{F}_{1}\right)\right]\right\} d S .
\end{aligned}
$$

One can check on the example $\mathcal{F}_{1}=T^{+} \mathcal{F}_{0}$ that condition (7.32) and thus (7.31) is satisfied not for all $\left(\mathcal{F}_{0}, \mathcal{F}_{1}\right) \in \mathcal{H}^{1,0}(\Omega ; A) \times H^{-\frac{1}{2}}(\partial \Omega)$.

Theorem 7.3. If conditions (4.4) and (4.10) hold, then the operator $\mathcal{M}^{21}: \mathbb{H} \rightarrow \mathbb{F}^{21}$ is a continuous Fredholm operator with zero index. It has one-dimensional null-space spanned over the element $\left(u^{0}, \psi^{0}, \varphi^{0}\right)$ defined in Theorem 6.2(i) and the cokernel spanned over the functional $g^{0}$ defined by (7.31). 
Proof. The claim about the null-space, particularly that its dimension is 1 , follows from Theorem 6.2(i).

Let now consider the equation $\mathcal{M}^{21} \mathcal{U}=\left(\mathcal{F}_{0}, \mathcal{F}_{1}\right)^{\top}$, i.e.,

$$
\begin{aligned}
u+\mathcal{R} u-V \psi+W \varphi & =\mathcal{F}_{0} \quad \text { in } \quad \Omega, \\
\frac{1}{2} \psi+T^{+} \mathcal{R} u-\mathcal{W}^{\prime} \psi+\mathcal{L}^{+} \varphi & =\mathcal{F}_{1} \quad \text { on } \quad \partial \Omega .
\end{aligned}
$$

with arbitrary $\left(\mathcal{F}_{0}, \mathcal{F}_{1}\right) \in \mathbb{F}^{21}$ for $(u, \psi, \varphi) \in \mathbb{H}$. By Corollary 7.2 , if $g^{0}\left(\mathcal{F}_{0}, \mathcal{F}_{1}\right)=0$, where the linear functional $g^{0} \in \mathbb{F}^{21 *}$ is defined in (7.31), then the right hand side is representable in form (7.29)-(7.30) and the equation is solvable due to Theorem 6.2(ii).

On the other hand, we have from (5.4), the jump Theorem 4.2 and Lemma 4.1,

$$
\begin{aligned}
& g^{0}\left(\mathcal{M}^{21}(u, \psi, \varphi)^{\top}\right)=\int_{\partial \Omega} T_{\Delta}^{+}\{a[u+\mathcal{R} u-V \psi+W \varphi \\
& \left.\left.\quad-V\left(T^{+}(u+\mathcal{R} u-V \psi+W \varphi)-\left(\frac{1}{2} \psi+T^{+} \mathcal{R} u-\mathcal{W}^{\prime} \psi+\mathcal{L}^{+} \varphi\right)\right)\right]\right\} d S \\
& =\int_{\partial \Omega} T_{\Delta}^{+}\left\{a\left[u+\mathcal{R} u+W \varphi-V T^{+} u\right]\right\} d S=\int_{\partial \Omega} T_{\Delta}^{+}\{a \mathcal{P} A u\} d S
\end{aligned}
$$

Since $u \in \mathcal{H}^{1,0}(\Omega ; A)$, by Theorem 4.1 we have $\mathcal{P} A u \in \mathcal{H}^{1,0}\left(\mathbb{R}^{3} ; A\right)$ and thus $a \mathcal{P} A u \in$ $\mathcal{H}^{1,0}\left(\mathbb{R}^{3} ; \Delta\right) \subset H_{\text {loc }}^{2}\left(\mathbb{R}^{3}\right)$. This implies that $T_{\Delta}^{+}\{a \mathcal{P} A u\}=T_{\Delta}^{-}\{a \mathcal{P} A u\}$ on $\partial \Omega$ and the last integral in (7.33) is zero because $a \mathcal{P} A u$ is harmonic in the bounded domain $\Omega^{-}$. Thus the range of the operator $\mathcal{M}^{21}: \mathbb{H} \rightarrow \mathbb{F}^{21}$ coincides with the elements of $\left(\mathcal{F}_{0}, \mathcal{F}_{1}\right) \in \mathbb{F}^{21}$ such that $g^{0}\left(\mathcal{F}_{0}, \mathcal{F}_{1}\right)=0$, which implies that the dimension of the coker $\mathcal{M}^{21}: \mathbb{H} \rightarrow \mathbb{F}^{21}$ is 1 . Since the dimension of the null-space is also 1, we conclude that the operators is Fredholm with zero index.

\subsection{Properties of operators $\mathcal{M}^{\alpha \beta}: \mathbb{X} \rightarrow \mathbb{Y}^{\alpha \beta}$}

To prove in [2] the invertibility of the counterparts of the operators $\mathcal{M}^{\alpha \beta}: \mathbb{X} \rightarrow \mathbb{Y}^{\alpha \beta}$ for bounded domains, we essentially used the compactness of the operator $\mathcal{R}: H^{1}(\Omega) \rightarrow$ $H^{1}(\Omega)$ based on the Rellich compactness theorem. However, the latter theorem does not hold for unbounded domains with compact boundaries, and to cope with this, we will split the operator $\mathcal{R}$ into two parts, one of which can be made arbitrarily small while the other one is compact, if the PDE coefficient satisfies the additional condition

$$
\lim _{x \rightarrow \infty} \rho(x) \nabla a(x)=0 .
$$

Lemma 7.4. Let conditions (4.4) and (7.34) hold. Then for any $\epsilon>0$ the operator $\mathcal{R}$ can be represented as $\mathcal{R}=\mathcal{R}_{c}+\mathcal{R}_{s}$, where $\left\|\mathcal{R}_{s}\right\|_{\mathcal{H}^{1}(\Omega) \rightarrow \mathcal{H}^{1}(\Omega)}<\epsilon$, while $\mathcal{R}_{c}: \mathcal{H}^{1}(\Omega) \rightarrow \mathcal{H}^{1}(\Omega)$ is compact.

Proof. Let $B_{\eta}$ be a ball centered at 0 with a radius $\eta$ such that $\partial \Omega \subset B_{\eta}$ and let $\mu \in \mathcal{D}\left(\mathbb{R}^{3}\right)$ be a cut-off function such that $\mu=1$ in $B_{\eta}, \mu=0$ in $\mathbb{R}^{3} \backslash B_{2 \eta}$ and $0 \leq \mu(x) \leq 1$ in $\mathbb{R}^{3}$. Denote $\mathcal{R}_{c} g:=\mathcal{R}(\mu g), \mathcal{R}_{s} g:=\mathcal{R}((1-\mu) g)$.

By (4.8) we have for arbitrary $g \in \mathcal{H}^{1}(\Omega)$,

$$
\left\|\mathcal{R}_{s} g\right\|_{\mathcal{H}^{1}(\Omega)}=\left\|\sum_{j=1}^{3} \mathcal{P} \partial_{j}\left[(1-\mu) g \partial_{j} a\right]\right\|_{\mathcal{H}^{1}(\Omega)} \leq Q\|\mathcal{P}\|_{\widetilde{\mathcal{H}}^{-1}(\Omega) \rightarrow \mathcal{H}^{1}(\Omega)}
$$


where

$$
\begin{aligned}
Q & :=\sum_{j=1}^{3}\left\|\partial_{j}\left[(1-\mu) g \partial_{j} a\right]\right\|_{\widetilde{\mathcal{H}}^{-1}(\Omega)} \leq \sum_{j=1}^{3}\left\|(1-\mu) g \partial_{j} a\right\|_{L_{2}(\Omega)} \\
& \leq 3\|g\|_{L_{2}\left(\rho^{-1} ; \Omega\right)}\|\rho \nabla a\|_{L_{\infty}\left(\mathbb{R}^{3} \backslash B_{\eta}\right)} \leq 3\|\rho \nabla a\|_{L_{\infty}\left(\mathbb{R}^{3} \backslash B_{\eta}\right)}\|g\|_{\mathcal{H}^{1}(\Omega)}
\end{aligned}
$$

Thus for the norm of the operator $\mathcal{R}_{s}$ we have,

$$
\left\|\mathcal{R}_{s}\right\|_{\mathcal{H}^{1}(\Omega) \rightarrow \mathcal{H}^{1}(\Omega)} \leq 3\|\rho \nabla a\|_{L_{\infty}\left(\mathbb{R}^{3} \backslash B_{\eta}\right)}\|\mathcal{P}\|_{\widetilde{\mathcal{H}}^{-1}(\Omega) \rightarrow \mathcal{H}^{1}(\Omega)} \rightarrow 0 \text { as } \eta \rightarrow \infty,
$$

as claimed.

Let us prove the claim about the operator $\mathcal{R}_{c}$. Since the support of $\mu$ belongs to $\overline{B_{2 \eta}}$, for any fixed $\eta$ the operator $\mathcal{R}_{c}: \mathcal{H}^{1}(\Omega) \rightarrow \mathcal{H}^{1}(\Omega)$ can be represented as $\mathcal{R}_{c} g=\mathcal{R}_{\Omega_{2 \eta}}\left[\mu r_{\Omega_{2 \eta}} g\right]$, where $\Omega_{2 \eta}=\Omega \bigcap B_{2 \eta}$ and the operator $\mathcal{R}_{\Omega_{2 \eta}}$ is given by the second relation in (4.6) with $\Omega$ replaced by $\Omega_{2 \eta}$. The operator $\mathcal{R}_{\Omega_{2 \eta}}: L_{2}\left(\Omega_{2 \eta}\right) \rightarrow \mathcal{H}^{1}(\Omega)$ is continuous by (4.13) since $L_{2}\left(\Omega_{2 \eta}\right)=L_{2}\left(\rho^{-1} ; \Omega_{2 \eta}\right)$ for the bounded domain $\Omega_{2 \eta}$. On the other hand, the restriction operator $r_{\Omega_{2 \eta}}: \mathcal{H}^{1}(\Omega) \rightarrow \mathcal{H}^{1}\left(\Omega_{2 \eta}\right)=H^{1}\left(\Omega_{2 \eta}\right)$ is continuous while the imbedding of $H^{1}\left(\Omega_{2 \eta}\right)$ in $L_{2}\left(\Omega_{2 \eta}\right)$ is compact, which implies that the operator $\mathcal{R}_{c}: \mathcal{H}^{1}(\Omega) \rightarrow \mathcal{H}^{1}(\Omega)$ is compact.

Lemma 7.4 implies the following corollary.

Corollary 7.3. Let conditions (4.4) and (7.34) hold. Then the operator $I+\mathcal{R}$ : $\mathcal{H}^{1}(\Omega) \rightarrow \mathcal{H}^{1}(\Omega)$ is Fredholm with zero index.

Proof. Representing $\mathcal{R}=\mathcal{R}_{c}+\mathcal{R}_{s}$ by Lemma 7.4 so that $\left\|\mathcal{R}_{s}\right\|_{\mathcal{H}^{1}(\Omega)}<1$ and $\mathcal{R}_{c}$ : $\mathcal{H}^{1}(\Omega) \rightarrow \mathcal{H}^{1}(\Omega)$ is compact, we obtain that $I+\mathcal{R}_{s}: \mathcal{H}^{1}(\Omega) \rightarrow \mathcal{H}^{1}(\Omega)$ is invertible, which implies the lemma claim.

Theorem 7.4. If conditions (4.4), (4.10) and (7.34) hold, then the operators

$$
\mathcal{M}^{11}: \mathbb{X} \rightarrow \mathbb{Y}^{11}, \quad \mathcal{M}^{12}: \mathbb{X} \rightarrow \mathbb{Y}^{12}, \quad \mathcal{M}^{22}: \mathbb{X} \rightarrow \mathbb{Y}^{22}
$$

are continuous and continuously invertible.

Proof. By the mapping properties of the potentials, operators (7.35) are continuous and we now prove their invertibility.

Invertibility of operator $\mathcal{M}^{11}$. Let us consider the operator

$$
\mathcal{M}_{0}^{11}: \mathbb{X} \rightarrow \mathbb{Y}^{11}
$$

where

$$
\mathcal{M}_{0}^{11}:=\left[\begin{array}{ccc}
I & -V & W \\
0 & -r_{\partial_{D} \Omega} \mathcal{V} & r_{\partial_{D} \Omega} \mathcal{W} \\
0 & 0 & r_{\partial_{N} \Omega} \hat{\mathcal{L}}
\end{array}\right],
$$

and $\hat{\mathcal{L}}$ is defined in (4.30). Evidently operator (7.36) is continuous. The diagonal operators of the triangular matrix operator $\mathcal{M}_{0}^{11}$ are continuously invertible (cf. the proof of $[2$, Theorem 5.3]), implying that the operator $\left(\mathcal{M}_{0}^{11}\right)^{-1}$ inverse to $(7.36)$ is continuous. 
Let us now represent $\mathcal{R}=\mathcal{R}_{s}+\mathcal{R}_{c}$ by Lemma 7.4 so that the operator $\mathcal{R}_{s}$ is sufficiently small for the operator

to satisfy the inequality

$$
\mathcal{M}_{s}^{11}:=\left[\begin{array}{ccc}
\mathcal{R}_{s} & 0 & 0 \\
r_{\partial_{D} \Omega} \gamma^{+} \mathcal{R}_{s} & 0 & 0 \\
r_{\partial_{N} \Omega} T^{+} \mathcal{R}_{s} & 0 & 0
\end{array}\right]
$$

$$
\left\|\mathcal{M}_{s}^{11}\right\|_{\mathbb{X} \rightarrow \mathbb{Y}^{11}}<1 /\left\|\left(\mathcal{M}_{0}^{11}\right)^{-1}\right\|_{\mathbb{Y}^{11} \rightarrow \mathbb{X}}
$$

Then the operator $M_{0}^{11}+\mathcal{M}_{s}^{11}: \mathbb{X} \rightarrow \mathbb{Y}^{11}$ is continuously invertible, while the operator $\mathcal{M}_{c}^{11}:=\mathcal{M}^{11}-\mathcal{M}_{0}^{11}-\mathcal{M}_{s}^{11}: \mathbb{X} \rightarrow \mathbb{Y}^{11}$ is compact by Lemma 7.4 and by the mapping properties of the operators $\mathcal{W}^{\prime}$ and $\mathcal{L}^{+}-\hat{\mathcal{L}}$, see [2, Theorems 3.4, 3.6]. This implies that operator $\mathcal{M}^{11}: \mathbb{X} \rightarrow \mathbb{Y}^{11}$ is a Fredholm operator with zero index. Since by Theorem 6.1 it is also injective, we conclude that it is invertible.

Invertibility of operator $\mathcal{M}^{\mathbf{1 2}}$. Let us consider the auxiliary operator

$$
\mathcal{M}_{0}^{12}:=\left[\begin{array}{ccc}
I & -V & W \\
0 & -\mathcal{V} & \frac{1}{2} I
\end{array}\right]: \mathbb{X} \rightarrow \mathbb{Y}^{12} .
$$

Evidently operator (7.37) is continuous. Any solution $\mathcal{U}=(u, \psi, \varphi)^{\top} \in \mathbb{X}$ of the equation $\mathcal{M}_{0}^{12} \mathcal{U}=\mathcal{F}$, where $\mathcal{F}=\left(\mathcal{F}_{0}, \mathcal{F}_{1}\right)^{\top} \in \mathcal{H}^{1}(\Omega) \times H^{\frac{1}{2}}(\partial \Omega)$ will solve also the following extended system of three equations,

$$
\begin{aligned}
u+W \varphi-V \psi & =\mathcal{F}_{0} \quad \text { in } \quad \Omega \\
\frac{1}{2} \varphi-\mathcal{V} \psi & =\mathcal{F}_{1} \quad \text { on } \quad \partial \Omega \\
-r_{\partial_{D} \Omega} \mathcal{V} \psi & =r_{\partial_{D} \Omega} \mathcal{F}_{1} \quad \text { on } \quad \partial_{D} \Omega
\end{aligned}
$$

and vice-versa. Taking into account that invertibility of the operator $r_{\partial_{D} \Omega} \mathcal{V}$ follows from the first relation in (4.27) and e.g. [27, Theorem 2.7(i)], the diagonal operators of the system,

$$
\begin{gathered}
I: \mathcal{H}^{1}(\Omega) \rightarrow \mathcal{H}^{1}(\Omega) \\
\frac{1}{2} I: H^{\frac{1}{2}}(\partial \Omega) \rightarrow H^{\frac{1}{2}}(\partial \Omega), \\
-r_{\partial_{D} \Omega} \mathcal{V}: \widetilde{H}^{-\frac{1}{2}}\left(\partial_{D} \Omega\right) \rightarrow H^{\frac{1}{2}}\left(\partial_{D} \Omega\right),
\end{gathered}
$$

are continuously invertible implying that the triangular matrix operator of the system is also invertible. If $\psi \in \widetilde{H}^{-\frac{1}{2}}\left(\partial_{D} \Omega\right)$ solves equation $(7.40)$, then $\varphi=2\left(\mathcal{F}_{1}+\mathcal{V} \psi\right) \in \widetilde{H}^{\frac{1}{2}}\left(\partial_{N} \Omega\right)$ by equation (7.39), and we arrive at invertibility of the operator (7.37). The rest of the proof for the operator $\mathcal{M}^{12}$ is similar to the one for $\mathcal{M}^{11}$.

Invertibility of operator $\mathcal{M}^{\mathbf{2 2}}$. Let us consider the auxiliary operator

$$
\mathcal{M}_{0}^{22}: \mathbb{X} \rightarrow \mathbb{Y}^{22}
$$

where

$$
\mathcal{M}_{0}^{22}:=\left[\begin{array}{ccc}
I & -V & W \\
0 & r_{\partial_{D} \Omega}\left(\frac{1}{2} I-\mathcal{W}^{\prime}{ }_{\Delta}\right) & r_{\partial_{D} \Omega} \hat{\mathcal{L}} \\
0 & -r_{\partial_{N} \Omega} \mathcal{V} & r_{\partial_{N} \Omega}\left(\frac{1}{2} I+\mathcal{W}\right)
\end{array}\right]
$$


Operator (7.41) is evidently continuous and can be considered as a matrix block-triangle operator with the lower diagonal block

$$
\hat{\mathcal{M}}_{0}^{22}:=\left[\begin{array}{cc}
r_{\partial_{D} \Omega}\left(\frac{1}{2} I-\mathcal{W}^{\prime}{ }_{\Delta}\right) & r_{\partial_{D} \Omega} \hat{\mathcal{L}} \\
-r_{\partial_{N} \Omega} \mathcal{V} & r_{\partial_{N} \Omega}\left(\frac{1}{2} I+\mathcal{W}\right)
\end{array}\right] .
$$

Taking into account relations (4.27) and (4.29), we can represent

$$
\hat{\mathcal{M}}_{0}^{22} g=\operatorname{diag}\left(1, \frac{1}{a}\right) \hat{\mathcal{M}}_{\Delta}^{22}[\operatorname{diag}(1, a) g],
$$

for any $g=\left(g_{1}, g_{2}\right)^{\top} \in \widetilde{H}^{-\frac{1}{2}}\left(\partial_{D} \Omega\right) \times \widetilde{H}^{\frac{1}{2}}\left(\partial_{N} \Omega\right)$, where $\operatorname{diag}(1,1 / a)$ and $\operatorname{diag}(1, a)$ are diagonal $2 \times 2$ matrices, while the operator $\hat{\mathcal{M}}_{\Delta}^{22}$ given by (7.19) is invertible by Theorem 7.2. Since $0<a_{0}<a(x)<a_{1}<\infty$, this implies the invertibility of the operator

$$
\hat{\mathcal{M}}_{0}^{22}: \widetilde{H}^{-\frac{1}{2}}\left(\partial_{D} \Omega\right) \times \widetilde{H}^{\frac{1}{2}}\left(\partial_{N} \Omega\right) \rightarrow H^{-\frac{1}{2}}\left(\partial_{D} \Omega\right) \times H^{\frac{1}{2}}\left(\partial_{N} \Omega\right)
$$

and thus of operator (7.41). The rest of the proof for the operator $\mathcal{M}^{22}$ is similar to the one for $\mathcal{M}^{11}$.

Theorem 7.5. If conditions (4.4), (4.10) and (7.34) hold, then the operator $\mathcal{M}^{21}: \mathbb{X} \rightarrow$ $\mathbb{Y}^{21}$ is a continuous Fredholm operator with zero index. It has one-dimensional null-space spanned over the element $\left(u^{0}, \psi^{0}, \varphi^{0}\right)$ defined in Theorem 6.2(i) and the cokernel spanned over the functional $g^{0}$ defined by (7.31).

Proof. The claim about the null-space, particularly that its dimension is 1 , follows from Theorem 6.2(i).

Let us consider the auxiliary operator

$$
\mathcal{M}_{0}^{21}:=\left[\begin{array}{ccc}
I & -V & W \\
0 & -\frac{1}{2} I & \hat{\mathcal{L}}
\end{array}\right]: \mathbb{X} \rightarrow \mathbb{Y}^{21} .
$$

Evidently operator (7.42) is continuous. Any solution $\mathcal{U}=(u, \psi, \varphi)^{\top} \in \mathbb{X}$ of the equation $\mathcal{M}_{0}^{21} \mathcal{U}=\mathcal{F}$, where $\mathcal{F}=\left(\mathcal{F}_{0}, \mathcal{F}_{1}\right)^{\top} \in \mathcal{H}^{1}(\Omega) \times H^{-\frac{1}{2}}(\partial \Omega)$ will also solve the following extended system of three equations,

$$
\begin{aligned}
u-V \psi+W \varphi & =\mathcal{F}_{0} \quad \text { in } \quad \Omega, \\
-\frac{1}{2} \psi+\hat{\mathcal{L}} \varphi & =\mathcal{F}_{1} \quad \text { on } \quad \partial \Omega, \\
r_{\partial_{N} \Omega} \hat{\mathcal{L}} \varphi & =r_{\partial_{N} \Omega} \mathcal{F}_{1} \quad \text { on } \quad \partial_{N} \Omega,
\end{aligned}
$$

and vice-versa. Taking into account that invertibility of the operator $r_{\partial_{N} \Omega} \hat{\mathcal{L}}$ follows from relation (4.30) and e.g. [27, Theorem 2.7(ii)], the diagonal operators of the system,

$$
\begin{gathered}
I: \mathcal{H}^{1}(\Omega) \rightarrow \mathcal{H}^{1}(\Omega) \\
\frac{1}{2} I: H^{-\frac{1}{2}}(\partial \Omega) \rightarrow H^{-\frac{1}{2}}(\partial \Omega), \\
r_{\partial_{N} \Omega} \hat{\mathcal{L}}: \widetilde{H}^{\frac{1}{2}}\left(\partial_{N} \Omega\right) \rightarrow H^{-\frac{1}{2}}\left(\partial_{N} \Omega\right),
\end{gathered}
$$

are continuously invertible implying that the triangular matrix operator of the system is also invertible. If $\varphi \in \widetilde{H}^{\frac{1}{2}}\left(\partial_{D} \Omega\right)$ solves the third equation of the system, then $\mathcal{F}_{1}-\hat{\mathcal{L}} \varphi \in$ 
$\widetilde{H}^{-\frac{1}{2}}\left(\partial_{N} \Omega\right)$, and we arrive at invertibility of the operator (7.42). Then the reasoning similar to the second paragraph of the proof for operator $\mathcal{M}^{11}$ in Theorem 7.4 implies that operator $\mathcal{M}^{21}: \mathbb{X} \rightarrow \mathbb{Y}^{21}$ is Fredholm with zero index.

To prove that the cokernel is spanned over the functional $g^{0}$ defined by (7.31), it suffice to prove that for any for any $\mathcal{U}=(u, \psi, \varphi)^{\top} \in \mathbb{X}$, the right hand side couple $\mathcal{F}^{21}=\left(\mathcal{F}_{0}, \mathcal{F}_{1}\right) \in \mathbb{Y}^{21}$ of the BDIE system $\mathcal{M}^{21} \mathcal{U}=\mathcal{F}^{21}$, satisfies condition (7.31). Let a sequence $u_{k} \in \mathcal{D}(\bar{\Omega}) \subset \mathcal{H}^{1}(\Omega), k=1, \ldots \infty$, converge to $u$ in $\mathcal{H}^{1}(\Omega)$ and denote $\mathcal{U}_{k}=\left(u_{k}, \psi, \varphi\right)^{\top}$. Then $\mathcal{M}^{21} \mathcal{U}_{k} \in \mathbb{F}^{21}$ by the mapping properties of the potentials and thus $g^{0}\left(\mathcal{M}^{21} \mathcal{U}_{k}\right)=0$ by Theorem 7.3. Since $M^{21}: \mathbb{X} \rightarrow \mathbb{Y}^{21}$ is a continuous operator and $g^{0}$ defined by $(7.31)$ is a continuous functional on $\mathbb{Y}^{21}$, we obtain that $g^{0}\left(\mathcal{M}^{21} \mathcal{U}_{k}\right)$ converges to $g^{0}\left(\mathcal{M}^{21} \mathcal{U}\right)$, i.e., $g^{0}\left(\mathcal{M}^{21} \mathcal{U}\right)=0$.

\section{Appendix: Variational BVP Settings}

Generalizing the proofs of $[24,11]$ for a general divergent-form elliptic equation in $\mathbb{R}^{n}$ and of $[8,13,7]$ for the Dirichlet and Neumann problems for the Poisson equation in an unbounded domain $\Omega$, we prove in this section unique solvability of the Dirichlet, Neumann and mixed boundary-value problems for variable-coefficient equation (2.1) in an unbounded domain $\Omega \subset \mathbb{R}^{n}$ with a compact Lipschitz boundary using their variational settings and the LaxMilgram lemma.

\subsection{Dirichlet problem}

Let us first reformulate the Dirichlet problem (3.5)-(3.6) with a more general right hand side $f$ in the following weak form.

$(D)$ : Given $\varphi_{0} \in H^{\frac{1}{2}}(\partial \Omega)$ and $f \in \mathcal{H}^{-1}(\Omega)$, find $u \in \mathcal{H}^{1}(\Omega)$ such that

$$
\begin{aligned}
\mathcal{E}(u, v) & =-\langle f, v\rangle_{\Omega} \quad \forall v \in \widetilde{\mathcal{H}}^{1}(\Omega), \\
\gamma^{+} u & =\varphi_{0} \quad \text { on } \quad \partial \Omega .
\end{aligned}
$$

Taking in mind that the space $H_{0}^{1}(\Omega)$ of functions $g \in H^{1}(\Omega)$ such that $\gamma^{+} g=0$ can be identified with the space $\tilde{g} \in \widetilde{H}^{1}(\Omega)$ (see e.g. [14, Theorems 3.33, 3.40]), one can easily prove that the space $\mathcal{H}_{0}^{1}(\Omega)$ of functions $g \in \mathcal{H}^{1}(\Omega)$ such that $\gamma^{+} g=0$ can be identified with the space $\widetilde{\mathcal{H}}^{1}(\Omega)$ with equivalent norms. Then problem (8.1)-(8.2) with the homogeneous Dirichlet condition, $\varphi_{0}=0$ is reduced to the following variational problem.

$\left(D_{0}\right)$ : Given $f \in \mathcal{H}^{-1}(\Omega)$, find $u \in \mathcal{H}_{0}^{1}(\Omega)$ such that $\mathcal{E}(u, v)=-\langle f, v\rangle_{\Omega} \quad \forall v \in \mathcal{H}_{0}^{1}(\Omega)$.

By (2.5) and the norm definitions (2.2) and (2.3), we have estimates

$$
\begin{aligned}
|\mathcal{E}(u, v)| & \leq a_{1}|u|_{\mathcal{H}^{1}(\Omega)}|v|_{\mathcal{H}^{1}(\Omega)} \leq a_{1}\|u\|_{\mathcal{H}^{1}(\Omega)}\|v\|_{\mathcal{H}^{1}(\Omega)}, \\
\mathcal{E}(u, u) & \geq a_{0}|u|_{\mathcal{H}^{1}(\Omega)}^{2} \geq C a_{0}\|u\|_{\mathcal{H}^{1}(\Omega)}^{2}
\end{aligned}
$$

implying the continuity on $\mathcal{H}^{1}(\Omega) \times \mathcal{H}^{1}(\Omega)$ and $\mathcal{H}^{1}(\Omega)$-ellipticity and thus the continuity on $\mathcal{H}_{0}^{1}(\Omega) \times \mathcal{H}_{0}^{1}(\Omega)$ and $\mathcal{H}_{0}{ }^{1}(\Omega)$-ellipticity of the bilinear functional $\mathcal{E}$. The estimate

$$
\left|\langle f, v\rangle_{\Omega}\right| \leq\|f\|_{\mathcal{H}^{-1}(\Omega)}\|v\|_{\widetilde{\mathcal{H}}^{1}(\Omega)} \leq C\|f\|_{\mathcal{H}^{-1}(\Omega)}\|v\|_{\mathcal{H}_{0}^{1}(\Omega)}
$$

implies the continuity of the functional $f$ on $\mathcal{H}_{0}^{1}(\Omega)$. Then due to the Lax-Milgram lemma, the problem $\left(D_{0}\right)$ is uniquely solvable and its solution is $u=\mathcal{A}_{D 0}^{-1} f$, where the operator $A_{D 0}^{-1}: \mathcal{H}^{-1}(\Omega) \rightarrow \mathcal{H}_{0}^{1}(\Omega)$ is continuous.

Let now $B$ be an open ball such that $\partial \Omega \subset B$ and denote $\Omega^{\prime}=\Omega \cap B$. Let us now look for a solution of the general Dirichlet problem (8.1)-(8.2) in the form $u=u_{0}+\tilde{u}_{1}$, where 
$\tilde{u}_{1} \in \mathcal{H}^{1}(\Omega)$ is the extension by zero to $\Omega$ of the solution $u_{1} \in H^{1}\left(\Omega^{\prime}\right)$ of the equation $A u_{1}=0$ in the bounded domain $\Omega^{\prime}$ with the Dirichlet conditions $\gamma^{+} u_{1}=\varphi_{0}$ on $\partial \Omega$, $\gamma^{+} u_{1}=0$ on $\partial B$. The mapping of $\varphi_{0} \in H^{\frac{1}{2}}(\partial \Omega)$ to $\tilde{u}_{1} \in \mathcal{H}^{1}(\Omega)$ is evidently continuous. Then $u_{0} \in \mathcal{H}_{0}^{1}(\Omega)$ is the (unique) solution of the problem $D_{0}$ with the modified right hand side $f_{0}=f-A \tilde{u} \in \mathcal{H}^{-1}(\Omega)$. Taking into account that the homogeneous problem $(D)$ has only the trivial solution due to the unique solvability of the problem $\left(D_{0}\right)$, we arrive at the following assertion.

Theorem 8.1. Under conditions (2.6) the problem (D) and the Dirichlet problem (3.5)(3.6) are uniquely solvable and their solutions can be written as $u=\mathcal{A}_{D}^{-1}\left(f, \varphi_{0}\right)^{\top}$, where the operators $\mathcal{A}_{D}^{-1}: \mathcal{H}^{-1}(\Omega) \times H^{\frac{1}{2}}(\partial \Omega) \rightarrow \mathcal{H}^{1}(\Omega)$ and $\mathcal{A}_{D}^{-1}: L_{2}(\rho ; \Omega) \times H^{\frac{1}{2}}(\partial \Omega) \rightarrow$ $\mathcal{H}^{1,0}(\Omega ; A)$ are continuous.

\subsection{Neumann problem}

Taking into account the first Green identity (2.8), it is easy to show that the Neumann problem (3.7)-(3.8) is equivalent to the following weak problem

$(N)$ : Given $\psi_{0} \in H^{-\frac{1}{2}}(\partial \Omega)$ and $f \in L_{2}(\rho ; \Omega)$, find $u \in \mathcal{H}^{1,0}(\Omega ; A)$ such that

$$
\mathcal{E}(u, v)=-\langle f, v\rangle_{\Omega}+\left\langle\psi_{0}, \gamma^{+} v\right\rangle_{\partial \Omega} \quad \forall v \in \mathcal{H}^{1}(\Omega) .
$$

We associate with equation (8.5) also the following variational problem (in a wider space).

$(\check{N}):$ For $\check{f} \in \widetilde{\mathcal{H}}^{-1}(\Omega)$ find $u \in \mathcal{H}^{1}(\Omega)$ such that

$$
\mathcal{E}(u, v)=-\langle\check{f}, v\rangle \quad \forall v \in \mathcal{H}^{1}(\Omega) .
$$

The estimates (8.3) and (8.4) imply the continuity and $\mathcal{H}^{1}(\Omega)$-ellipticity of the bilinear functional $\mathcal{E}$, while the functional $\check{f} \in \widetilde{\mathcal{H}}^{-1}(\Omega)$ is continuous on $\mathcal{H}^{1}(\Omega)$ by the definition of the space $\widetilde{\mathcal{H}}^{-1}(\Omega)$. Then due to the Lax-Milgram lemma we arrive at the following assertion.

Theorem 8.2. Under conditions (2.6) the problem $(\check{N})$ is uniquely solvable and its solution is $u=\mathcal{A}_{\tilde{N}}^{-1} \check{f}$, where the operator $\mathcal{A}_{\check{N}}^{-1}: \widetilde{\mathcal{H}}^{-1}(\Omega) \rightarrow \mathcal{H}^{1}(\Omega)$ is continuous.

For problem $(N)$, let us define $\check{f}$ as $\langle\check{f}, v\rangle_{\Omega}=\langle f, v\rangle_{\Omega}-\left\langle\psi_{0}, \gamma^{+} v\right\rangle_{\partial \Omega} \quad \forall v \in \mathcal{H}^{1}(\Omega)$. Then the estimates

$$
\begin{aligned}
\left|\langle f, v\rangle_{\Omega}\right| & \leq\|f\|_{L_{2}(\rho ; \Omega)}\|v\|_{\mathcal{H}^{1}(\Omega)}, \\
\left|\left\langle\psi_{0}, \gamma^{+} v\right\rangle_{\partial \Omega}\right| & \leq\left\|\psi_{0}\right\|_{H^{-\frac{1}{2}}(\partial \Omega)}\left\|\gamma^{+}\right\|_{\mathcal{H}^{-1}(\Omega) \rightarrow H^{\frac{1}{2}}(\partial \Omega)}\|v\|_{\mathcal{H}^{1}(\Omega)}
\end{aligned}
$$

imply $\check{f} \in \widetilde{\mathcal{H}}^{-1}(\Omega)$ and we obtain the following corollary from Theorem 8.2.

Theorem 8.3. Under conditions (2.6) the problem $(N)$ and thus the Neumann problem (3.7)-(3.8) are uniquely solvable and their solution is $u=\mathcal{A}_{N}^{-1}\left(f, \psi_{0}\right)^{\top}$, where the operator $\mathcal{A}_{N}^{-1}: L_{2}(\rho ; \Omega) \times H^{-\frac{1}{2}}(\partial \Omega) \rightarrow \mathcal{H}^{1,0}(\Omega ; A)$ is continuous. 


\subsection{Mixed problem}

Due to the first Green identity (2.8), it is easy to show that the mixed problem (3.1)-(3.3) is equivalent to the following weak problem

$(M):$ Given $\varphi_{0} \in H^{\frac{1}{2}}\left(\partial_{D} \Omega\right), \psi_{0} \in H^{-\frac{1}{2}}\left(\partial_{N} \Omega\right)$ and $f \in L_{2}(\rho ; \Omega)$, find $u \in \mathcal{H}^{1,0}(\Omega ; A)$ such that

$$
\begin{aligned}
\mathcal{E}(u, v) & =-\langle f, v\rangle_{\Omega}+\left\langle\psi_{0}, \gamma^{+} v\right\rangle_{\partial_{N} \Omega} \quad \forall v \in \mathcal{H}_{0}^{1}\left(\Omega ; \partial_{D} \Omega\right) \\
\gamma^{+} u & =\varphi_{0} \quad \text { on } \quad \partial_{D} \Omega
\end{aligned}
$$

where $\mathcal{H}_{0}^{1}\left(\Omega ; \partial_{D} \Omega\right):=\left\{w \in \mathcal{H}^{1}(\Omega): \gamma^{+} w=0\right.$ on $\left.\partial_{D} \Omega\right\}$.

Let $\left[\mathcal{H}_{0}^{1}\left(\Omega ; \partial_{D} \Omega\right)\right]^{*}$ denote the space dual to $\mathcal{H}_{0}^{1}\left(\Omega ; \partial_{D} \Omega\right)$. We associate with the problem $(M)$ also the following weak problem (in a wider space).

$(\check{M})$ : Given $\varphi_{0} \in H^{\frac{1}{2}}\left(\partial_{D} \Omega\right)$ and $\check{f} \in\left[\mathcal{H}_{0}^{1}\left(\Omega ; \partial_{D} \Omega\right)\right]^{*}$, find $u \in \mathcal{H}^{1}(\Omega)$ such that

$$
\begin{aligned}
\mathcal{E}(u, v) & =-\langle\check{f}, v\rangle_{\Omega} \quad \forall v \in \mathcal{H}_{0}^{1}\left(\Omega ; \partial_{D} \Omega\right), \\
\gamma^{+} u & =\varphi_{0} \quad \text { on } \quad \partial_{D} \Omega .
\end{aligned}
$$

Then its special case for the homogeneous Dirichlet condition, $\varphi_{0}=0$ on $\partial_{D} \Omega$, reduces to the variational problem

$\left(\check{M}_{0}\right):$ Given $\check{f} \in\left[\mathcal{H}_{0}^{1}\left(\Omega ; \partial_{D} \Omega\right)\right]^{*}$, find $u \in \mathcal{H}_{0}^{1}\left(\Omega ; \partial_{D} \Omega\right)$ such that

$$
\mathcal{E}(u, v)=-\langle\check{f}, v\rangle \quad \forall v \in \mathcal{H}_{0}^{1}(\Omega ; \partial \Omega)
$$

Since the norm in $\mathcal{H}_{0}^{1}\left(\Omega ; \partial_{D} \Omega\right)$ is induced by the norm in $\mathcal{H}^{1}(\Omega)$, the estimates $(8.3)$ and (8.4) imply the continuity on $\mathcal{H}_{0}^{1}\left(\Omega ; \partial_{D} \Omega\right) \times \mathcal{H}_{0}^{1}\left(\Omega ; \partial_{D} \Omega\right)$ and $\mathcal{H}_{0}^{1}\left(\Omega ; \partial_{D} \Omega\right)$-ellipticity of the bilinear functional $\mathcal{E}$. The functional $\check{f} \in\left[\mathcal{H}_{0}^{1}\left(\Omega ; \partial_{D} \Omega\right)\right]^{*}$ is evidently continuous on $\mathcal{H}_{0}^{1}\left(\Omega ; \partial_{D} \Omega\right)$. Then the Lax-Milgram lemma gives the following assertion.

Theorem 8.4. Under conditions (2.6) the problem $\left(\check{M}_{0}\right)$ is uniquely solvable and its solution is $u=\mathcal{A}_{\check{M}_{0}}^{-1} \check{f}$, where the operator $\mathcal{A}_{\check{M}_{0}}^{-1}:\left[\mathcal{H}_{0}^{1}\left(\Omega ; \partial_{D} \Omega\right)\right]^{*} \rightarrow \mathcal{H}_{0}^{1}\left(\Omega ; \partial_{D} \Omega\right)$ is continuous.

Let now $B$ be an open ball such that $\partial \Omega \subset B$ and denote $\Omega^{\prime}=\Omega \cap B$. Let $e$ : $H^{\frac{1}{2}}\left(\partial_{D} \Omega\right) \rightarrow H^{\frac{1}{2}}(\partial \Omega)$ be a linear continuous extension operator. Let us now look for a solution of the problem $(\check{M})$ in the form $u=u_{0}+\tilde{u}_{1}$, where $\tilde{u}_{1} \in \mathcal{H}^{1}(\Omega)$ is the extension by zero to $\Omega$ of the solution $u_{1} \in H^{1}\left(\Omega^{\prime}\right)$ of the equation $A u_{1}=0$ in the bounded domain $\Omega^{\prime}$ with the Dirichlet conditions $\gamma^{+} u_{1}=e \varphi_{0}$ on $\partial \Omega, \gamma^{+} u_{1}=0$ on $\partial B$. The mapping of $\varphi_{0} \in H^{\frac{1}{2}}(\partial \Omega)$ to $\tilde{u}_{1} \in \mathcal{H}^{1}(\Omega)$ is evidently continuous.

The operator $\check{A}_{\partial_{D} \Omega}: \mathcal{H}^{1}(\Omega) \rightarrow\left[\mathcal{H}^{1}\left(\Omega ; \partial_{D} \Omega\right)\right]^{*}$, defined as

$$
\left\langle\check{A}_{\partial_{D} \Omega} u, v\right\rangle_{\Omega}:=-\mathcal{E}(u, v) \quad \forall u \in \mathcal{H}^{1}(\Omega), \quad v \in \mathcal{H}^{1}\left(\Omega ; \partial_{D} \Omega\right),
$$

is bounded, cf. [18, Section 3]. This implies $\breve{A}_{\partial_{D} \Omega} \tilde{u}_{1} \in\left[\mathcal{H}^{1}\left(\Omega ; \partial_{D} \Omega\right)\right]^{*}$.

Then $u_{0} \in \mathcal{H}_{0}^{1}\left(\Omega ; \partial_{D} \Omega\right)$ is the (unique) solution of the problem $\check{M}_{0}$ with the modified right hand side $\widetilde{f}_{0}=\check{f}-\breve{A}_{\partial_{D} \Omega} \tilde{u}_{1} \in\left[\mathcal{H}^{1}\left(\Omega ; \partial_{D} \Omega\right)\right]^{*}$. Taking into account that by Theorem 8.4 the homogeneous problem $\left(\check{M}_{0}\right)$ and thus $(\check{M})$ has only the trivial solution, we arrive at the following assertion.

Theorem 8.5. Under conditions (2.6) the problem $(\check{M})$ is uniquely solvable and its solution is $u=\mathcal{A}_{\check{M}}^{-1}\left(\check{f}, \varphi_{0}\right)^{\top}$, where the operator $\mathcal{A}_{\check{M}}^{-1}:\left[\mathcal{H}_{0}^{1}\left(\Omega ; \partial_{D} \Omega\right)\right]^{*} \times H^{-\frac{1}{2}}(\partial \Omega) \rightarrow \mathcal{H}^{1}(\Omega)$ is continuous. 
For problem $(M)$, let us define $\check{f}$ as $\langle\check{f}, v\rangle_{\Omega}=\langle f, v\rangle_{\Omega}-\left\langle\psi_{0}, \gamma^{+} v\right\rangle_{\partial_{N} \Omega} \quad \forall v \in$ $\mathcal{H}_{0}^{1}\left(\Omega ; \partial_{D} \Omega\right)$. Then estimate (8.6) along with the estimate

$$
\left|\left\langle\psi_{0}, \gamma^{+} v\right\rangle_{\partial_{N} \Omega}\right| \leq\left\|\psi_{0}\right\|_{H^{-\frac{1}{2}}\left(\partial_{N} \Omega\right)}\left\|\gamma^{+}\right\|_{\mathcal{H}_{0}^{1}\left(\Omega ; \partial_{D} \Omega\right) \rightarrow \widetilde{H}^{\frac{1}{2}}\left(\partial_{N} \Omega\right)}\|v\|_{\mathcal{H}_{0}^{1}\left(\Omega ; \partial_{D} \Omega\right)}
$$

imply $\check{f} \in\left[\mathcal{H}_{0}^{1}\left(\Omega ; \partial_{D} \Omega\right)\right]^{*}$ and we obtain the following corollary from Theorem 8.5.

Theorem 8.6. Under conditions (2.6) the problem $(M)$ and thus the mixed problem (3.1)(3.3) are uniquely solvable and their solution is $u=\mathcal{A}_{M}^{-1}\left(f, \varphi_{0}, \psi_{0}\right)^{\top}$, where the operator $\mathcal{A}_{M}^{-1}: L_{2}(\rho ; \Omega) \times H^{\frac{1}{2}}\left(\partial_{D} \Omega\right) \times H^{-\frac{1}{2}}\left(\partial_{N} \Omega\right) \rightarrow \mathcal{H}^{1,0}(\Omega ; A)$ is continuous.

Remark that Theorems 8.3 and 8.4 give unique solvability of the generalized (aggregate) settings of, respectively, the Neumann and mixed problems in unbounded domains, that deal with the case when $f \in \mathcal{H}^{-1}(\Omega)$, which implies that the canonical co-normal derivative (2.7) is not well defined, while the corresponding generalized co-normal derivative is inherently non-unique, cf. [18, Section 3.2].

\section{Concluding Remarks}

Four different segregated direct boundary-domain integral equation systems, associated with the mixed (Dirichlet-Neumann) BVP for a scalar "Laplace" PDE with variable coefficient on a three-dimensional unbounded domain, have been formulated and analyzed in the paper. Equivalence of three of the BDIE systems to the original BVPs was proved in the case when right-hand side of the PDE is from $L_{2}(\rho ; \Omega)$, and the Dirichlet and the Neumann data are from the spaces $H^{\frac{1}{2}}\left(\partial_{D} \Omega\right)$ and $H^{-\frac{1}{2}}\left(\partial_{N} \Omega\right)$, respectively. The invertibility of the BDIE operators of these three systems was proved in the corresponding weighted Sobolev spaces. Fredholm properties of the fourth system were studied as well. This analysis was based on the invertibility in the weighted Sobolev spaces of the variable-coefficient BVPs in unbounded domains also proved in the paper.

Using the approach of [17], the united direct boundary-domain integro-differential systems can be also formulated and analyzed for the BVPs in exterior domains. The approach can be extended also to more general PDEs and to systems of PDEs, while smoothness of the boundary can be essentially relaxed, and the PDE right hand side can be considered in more general spaces, cf. [16].

Employing methods of [3], one can consider also the localized counterparts of the BDIEs for BVPs in exterior domains.

\section{Acknowledgments}

The work was supported by the grant EP/H020497/1 "Mathematical analysis of localised boundary-domain integral equations for BVPs with variable coefficients" of the EPSRC, UK.

\section{References}

[1] C. Amrouche, V. Girault and J. Giroire, Dirichlet and Neumann exterior problems for the n-dimensional Laplace operator. An approach in weighted Sobolev spaces, $J$. Math. Pures Appl. 76 (1997) 55-81.

[2] O. Chkadua, S. E. Mikhailov and D. Natroshvili, Analysis of direct boundary-domain integral equations for a mixed BVP with variable coefficient, I: Equivalence and invertibility, Journal of Integral Equations and Applications 21 (2009)(4) 499-543. 
[3] O. Chkadua, S. E. Mikhailov and D. Natroshvili, Analysis of some localized boundarydomain integral equations, Journal of Integral Equations and Applications 21 (2009) 405-445.

[4] O. Chkadua, S. E. Mikhailov and D. Natroshvili, Analysis of segregated boundarydomain integral equations for variable-coefficient problems with cracks, $\mathrm{Nu}$ merical Methods for Partial Differential Equations 27 (2011)(1) 121-140, doi: 10.1002/num.20639.

[5] M. Costabel, Boundary integral operators on Lipschitz domains: elementary results, SIAM J. Math. Anal. 19 (1988) 613-626.

[6] R. Dautray and J. Lions, Mathematical Analysis and Numerical Methods for Science and Technology, vol. 4: Integral Equations and Numerical Methods (Springer, BerlinHeidelberg-New York, 1990), ISBN 3-540-50209-2.

[7] J. Giroire, Étude de quelques problèmes aux limites extérieurs et résolution par équations intégrales, Thése de Doctorat d'État, Université Pierre-et-Marie-Curie (Paris-VI) (1987).

[8] J. Giroire and J. Nedelec, Numerical solution of an exterior Neumann problem using a double layer potential, Mathematics of Computation 32 (1978) 973-990.

[9] P. Grisvard, Elliptic Problems in Nonsmooth Domains (Pitman, Boston-LondonMelbourne, 1985), ISBN 0-273-08647-2.

[10] R. Grzhibovskis, S. E. Mikhailov and S. Rjasanow, Numerics of boundary-domain integral and integro-differential equations for BVP with variable coefficient in 3D, in IABEM 2011, Symposium of the International Association for Boundary Element Methods, 5-8 September 2011. Extended Abstracts (Brescia, Italy, 2011), pp. 163-168.

[11] B. Hanouzet, Espaces de Sobolev avec poids application au probleme de Dirichlet dans un demi espace, Rend. del Sem. Mat. della Univ. di Padova XLVI (1971) 227272.

[12] J.-L. Lions and E. Magenes, Non-Homogeneous Boundary Value Problems and Applications, vol. 1 (Springer, Berlin - Heidelberg - New York, 1972), ISBN 3-540-05363-8.

[13] J. Mäulen, Lösungen der Poissongleichung und harmonishe Vektorfelder in unbeshränkten Gebieten, Math. Meth. in the Appl. Sci. 5 (1983) 233-255.

[14] W. McLean, Strongly Elliptic Systems and Boundary Integral Equations (Cambridge University Press, Cambridge, UK, 2000), ISBN 0-521-66375 X.

[15] S. E. Mikhailov, Localized boundary-domain integral formulations for problems with variable coefficients, Engineering Analysis with Boundary Elements 26 (2002) 681690.

[16] S. E. Mikhailov, Analysis of extended boundary-domain integral and integrodifferential equations of some variable-coefficient BVP, in Advances in Boundary Integral Methods - Proceedings of the 5th UK Conference on Boundary Integral Methods, ed. K. Chen (University of Liverpool Publ., Liverpool, UK, 2005), ISBN 0 906370396 , pp. 106-125.

[17] S. E. Mikhailov, Analysis of united boundary-domain integro-differential and integral equations for a mixed BVP with variable coefficient, Math. Methods in Applied Sciences 29 (2006) 715-739.

[18] S. E. Mikhailov, Traces, extensions and co-normal derivatives for elliptic systems on Lipschitz domains, J. Math. Analysis and Appl. 378 (2011) 324-342, doi: 10.1016/j.jmaa.2010.12.027.

[19] S. E. Mikhailov and N. A. Mohamed, Iterative solution of boundary-domain integral equation for BVP with variable coefficient, in Proceedings of the 8th UK Conference on Boundary Integral Methods, ed. D. Lesnic (Leeds University Press, Leeds, UK, 2011), ISBN 978085316 2957, pp. 127-134. 
[20] S. E. Mikhailov and I. S. Nakhova, Mesh-based numerical implementation of the localized boundary-domain integral equation method to a variable-coefficient Neumann problem, J. Engineering Math. 51 (2005) 251-259.

[21] C. Miranda, Partial Differential Equations of Elliptic Type, 2-nd edn. (Springer, Berlin - Heidelberg - New York, 1970).

[22] J. Nedelec and J. Planchard, Une méthode variationelle d'éléments finis pour la résolution numérique d'un probléme extérieur dans $\mathbb{R}^{3}, R A I R O \quad 7$ (1973)(R3) 105129.

[23] J.-C. Nédélec, Acoustic and electromagnetic equations, vol. 144 of Applied Mathematical Sciences (Springer-Verlag, New York, 2001), ISBN 0-387-95155-5.

[24] J. B. Neto, Inhomogeneous boundary value problems in a half space, Ann. Sc. Sup. Pisa 19 (1965) 331-365.

[25] J. Sladek, V. Sladek and S. N. Atluri, Local boundary integral equation (LBIE) method for solving problems of elasticity with nonhomogeneous material properties, Comput. Mech. 24 (2000) 456-462.

[26] J. Sladek, V. Sladek and J.-D. Zhang, Local integro-differential equations with domain elements for the numerical solution of partial differential equations with variable coefficients, J. Eng. Math. 51 (2005) 261-282.

[27] E. Stephan, Boundary integral equations for screen problems in $\mathbb{R}^{3}$, Integral Equations and Operator Theory 10 (1987) 236-257.

[28] A. E. Taigbenu, The Green element method (Kluwer, Boston, 1999).

[29] T. Zhu, J.-D. Zhang and S. N. Atluri, A local boundary integral equation (LBIE) method in computational mechanics, and a meshless discretization approach, Computational Mechanics 21 (1998) 223-235.

[30] T. Zhu, J.-D. Zhang and S. N. Atluri, A meshless numerical method based on the local boundary integral equation (LBIE) to solve linear and non-linear boundary value problems, Engng. Anal. Bound. Elem. 23 (1999) 375-389. 